\title{
Local and global instabilities of flow in a flexible-walled channel
}

\author{
Peter S. Stewart ${ }^{\mathrm{a}}$, Sarah L. Waters ${ }^{\mathrm{b}}$, Oliver E. Jensen ${ }^{\mathrm{a}, *}$ \\ a School of Mathematical Sciences, University of Nottingham, University Park, Nottingham, NG7 2RD, UK \\ b Oxford Centre for Industrial and Applied Mathematics, University of Oxford, 24-29 St. Giles', Oxford, OX1 3LB, UK
}

\section{A R T I C L E I N F O}

\section{Article history:}

Received 30 May 2008

Received in revised form 28 November 2008

Accepted 9 March 2009

Available online $\mathrm{xxxx}$

\section{Keywords:}

Compliant channel

Convective instabilities

Wave-reflections

Self-excited oscillations

\begin{abstract}
A B S T R A C T
We consider laminar high-Reynolds-number flow through a long finite-length planar channel, where a segment of one wall is replaced by a massless membrane held under longitudinal tension. The flow is driven by a fixed pressure difference across the channel and is described using an integral form of the unsteady boundary-layer equations. The basic flow state, for which the channel has uniform width, exhibits static and oscillatory global instabilities, having distinct modal forms. In contrast, the corresponding local problem (neglecting boundary conditions associated with the rigid parts of the system) is found to be convectively, but not absolutely, unstable to small-amplitude disturbances in the absence of wall damping. We show how amplification of the primary global oscillatory instability can arise entirely from wave reflections with the rigid parts of the system, involving interacting travellingwave flutter and static-divergence modes that are convectively stable; alteration of the mean flow by oscillations makes the onset of this primary instability subcritical. We also show how distinct mechanisms of energy transfer differentiate the primary global mode from other modes of oscillatory instability.
\end{abstract}

(C) 2009 Elsevier Masson SAS. All rights reserved.

\section{Introduction}

Self-excited oscillations can arise when a flow is driven sufficiently rapidly through a flexible-walled tube. These oscillations have numerous physiological applications, including Korotkoff sound generation in sphygmomanometry and wheezing in lung airways (Heil and Jensen [1]; Grotberg and Jensen [2]). The primary experimental apparatus used in their investigation has been the Starling Resistor, in which a high-Reynolds-number flow is driven through a segment of elastic tube, which is mounted between two rigid tubes and is externally pressurised. The tube collapses nonaxisymmetrically and the internal flow, which may be turbulent, typically separates beyond the primary constriction in the tube. The resulting oscillations have attracted considerable attention because a complete explanation of their underlying mechanism (or mechanisms) remains elusive. The unsteady three-dimensional flow-structure interaction is a significant computational challenge, and the oscillations can exhibit considerable dynamical and fluidmechanical complexity $[3,4]$.

Theoretical studies of flow instabilities in flexible-walled channels or tubes have largely followed two routes: models of local instabilities in homogeneous domains; and models of global instabilities in bounded or non-uniform systems. The former investigations (reviewed by Davies and Carpenter [5]; Davies [6])

\footnotetext{
* Corresponding author.

E-mail address: oliver.jensen@nottingham.ac.uk (O.E. Jensen).
}

concern the interaction between hydrodynamic instabilities (modified Tollmien-Schlichting waves) and flow-induced surface instabilities (travelling-wave flutter (TWF) and static-divergence), but do not generally account for wave reflections at boundaries. The latter class of models address experimental configurations more directly, treating either the Starling Resistor or the simpler twodimensional analogue introduced by Pedley [7] (and considered below), in which a finite-length plane channel has a segment of one wall replaced by a membrane under longitudinal tension. Models of oscillations in the Starling Resistor system have developed from lumped-parameter representations $[8,9]$ to spatially one-dimensional models employing an ad hoc description of flow dissipation and tube wall mechanics [10-16]. These models incorporate the basic elements required to describe self-excited oscillation: fluid inertia, both in the collapsible segment and in the upstream and downstream rigid channels; a source of energy (provided by the mean flow); a restoring force (tube elasticity); and viscous dissipation (either distributed frictional losses or pressure losses associated with flow separation). By incorporating longitudinal tension, such models predict multiple modes of oscillations with frequencies falling in distinct bands, as seen experimentally by Bertram et al. [3] and in numerical simulations [17,18]; mode interactions can then lead to complex dynamical behaviour $[12,13]$.

More recently, Jensen and Heil [19] identified a mechanism for the generation of long-wavelength, high-frequency oscillations by asymptotic analysis and direct simulation of the two-dimensional channel system. They showed how normal modes of the system 
could be destabilised by the mean flow under suitable conditions. Self-excited oscillations grow by extracting kinetic energy from the mean flow, with exactly two thirds of the net kinetic energy flux dissipated by the oscillations and the remainder balanced by increased dissipation in the mean flow. Jensen and Heil [19] found that a necessary condition for instability was an asymmetry in the normal mode (associated with differences in the lengths of the upstream and downstream rigid channel segments), allowing kinetic energy fluxes at the upstream end of the collapsible segment to exceed those at the downstream end. This mechanism can in principle be generalised to three dimensions [20-23]. Study of the three-dimensional Starling-Resistor problem treating the fluid and solid mechanics exactly has so far been limited to steady flow (Hazel and Heil [24]; Marzo et al. [25]), although the first unsteady three-dimensional fluid-structure interaction calculations are now emerging [26].

Numerical solutions of the Navier-Stokes equations for the twodimensional channel problem (with Reynolds numbers in the midhundreds) have proved successful in capturing many important features of self-excited oscillations, in particular the generation of so-called vorticity waves (long-wavelength Tollmien-Schlichting waves) downstream of the oscillating segment $[17,18,27,28]$. However, despite this significant progress, the links between the local and global modes of instability have yet to be understood fully. Important questions to be answered concern the link between instabilities that are absolutely or convectively unstable in unbounded domains, and those that lead to growing global modes in a bounded system; whether vorticity waves generate, or are generated by, oscillations of the channel wall; and to what extent the mechanism of instability identified in [19] is relevant to the full range of oscillations that have been described. Simplified asymptotic models may provide a route to such understanding.

This paper seeks to shed light on two of these questions in the context of the two-dimensional channel system illustrated in Fig. 1 below and described in Section 2. A model is developed based on the following assumptions. First, an external pressure distribution is imposed outside the membrane which enables the system to support uniform Poiseuille flow with zero membrane displacement. This not only simplifies stability calculations, but reduces the influence of flow reversal or flow separation beyond any constriction in the channel. Second, the membrane, and the adjacent channel segments, are assumed long compared to the channel width, allowing a long-wavelength approximation to be exploited, reducing the governing Navier-Stokes equations to the boundarylayer equations. Third, the Reynolds number is assumed to be large enough for inertial forces to balance viscous forces in the core of the flow, but not so large that the flow forms viscous boundary layers or undergoes transition to turbulence. Fourth, to close the system within a one-dimensional framework, the velocity profile is assumed to be everywhere parabolic. This last assumption, which is widely used in related problems (such as the flow of thin liquid films down slopes) is fully justifiable for low Reynolds numbers, is in good agreement with Navier-Stokes simulations at moderate Reynolds numbers but is inaccurate at large Reynolds numbers or when the channel width varies rapidly with distance or time (Salamon [29]). Although ad hoc, its great advantage is that it provides a tractable model that provides an overall qualitative view of parameter space that is not presently feasible with larger-scale computational studies. However, this approach does not capture the full range of potential instabilities, failing in particular to describe Tollmien-Schlichting waves.

In Section 3 we consider the linear stability of the basic flow state, for which the channel has uniform width, by initially constructing global instabilities as a superposition of normal modes. In the large-tension limit we identify a set of oscillatory modes sustained by a balance between unsteady inertia and the restoring force of membrane tension (through the mechanism identified by Jensen and Heil [19]). In Section 4 we consider the corresponding local stability problem for interaction between the flow and the membrane, neglecting boundary conditions associated with the rigid parts of the system. We examine the absolute/convective stability of the basic flow state $[30,31]$ and distinguish the underlying modes using a framework based on activation energy [32-34]. This information is employed to deduce the relative contributions of wave propagation along the collapsible segment and reflections at the boundaries in generating a global instability. Wave reflections are shown to be essential in sustaining the primary high-frequency global mode. In Section 5 we use numerical simulations of the full non-linear system to describe the energy budget of different modes of oscillation, revealing differences that suggest the instability mechanism identified in [19] is not universal.

\section{Model}

We consider a planar channel of total length $L_{0}$, one wall of which contains a segment of thin elastic membrane under longitudinal tension. The channel walls elsewhere are rigid. When the membrane is flat, the channel has uniform width $a$ and the membrane has tension $T_{0}$ and damping coefficient $d_{0}$. Fluid of viscosity $\mu$ and constant density $\rho$ is driven through the channel by an imposed pressure drop. We set the pressure to be zero at the channel exit and impose constant upstream pressure $p_{0}$ at the channel entrance.

Introducing a velocity scale $U_{0}=p_{0} a^{2} /\left(12 \mu L_{0}\right)$, we scale all lengths on $a$ and time on $a / U_{0}$. We introduce Cartesian coordinates $(x, y)$ with origin $O$ (see Fig. 1 ) so that the channel walls lie at $y=0$ and $y=1$ (where the channel walls are rigid, that is for $-L_{1} \leqslant x<0$ and $L<x \leqslant L+L_{2}$ ), or $y=0$ and $y=h(x, t)$ (where the channel is deformable, for $0 \leqslant x \leqslant L)$. Here $h(0, t)=h(L, t)=1$ for all time $t$, and $a\left(L_{1}+L+L_{2}\right)=L_{0}$.

Scaling pressures on $\rho U_{0}^{2}$, the flow in the channel is governed by the dimensionless Navier-Stokes equations

$\nabla \cdot \mathbf{u}=0, \quad \frac{\mathrm{D} \mathbf{u}}{\mathrm{D} t}=-\nabla p+\frac{1}{R} \nabla^{2} \mathbf{u}$,

where the Reynolds number $R=\rho a U_{0} / \mu, \mathbf{u}=(u, v)$ is the velocity field and $p$ is the pressure. We impose constant upstream pressure $p=p_{u}=p_{0} /\left(\rho U_{0}^{2}\right)$ at $x=-L_{1}$. When $h=1$, the system admits steady, uniform, unit-flux Poiseuille flow along the channel, with

$$
\begin{aligned}
& \mathbf{u}=(6 y(1-y), 0), \quad p=p_{u}-\frac{12\left(L_{1}+x\right)}{R}, \\
& p_{u}=\frac{12\left(L_{1}+L_{2}+L\right)}{R}
\end{aligned}
$$

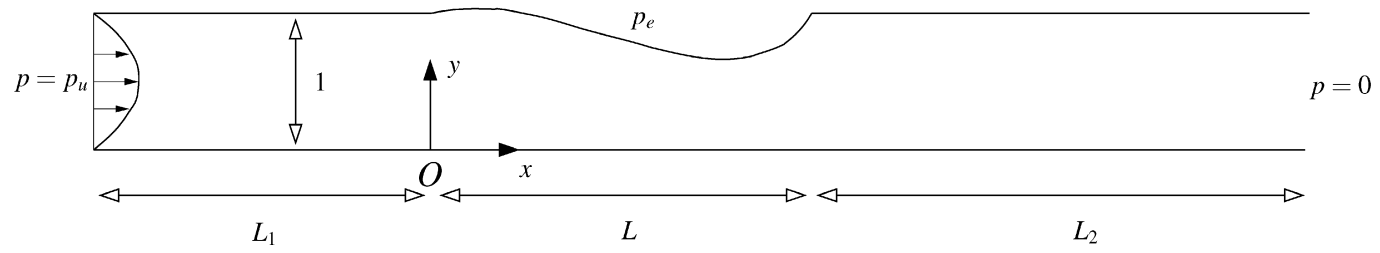

Fig. 1. The flow domain, showing dimensionless variables and parameters. 
If we impose the non-uniform external pressure distribution

$p_{e}(x)=p_{u}-\frac{12\left(L_{1}+x\right)}{R} \quad(0 \leqslant x \leqslant L)$,

then when $h=1$ there is no pressure difference either side of the membrane.

\subsection{Long-wave limit}

Suppose now that all three segments of the channel are long compared to the channel width. Defining $\epsilon=1 / L \ll 1$, we make a long-wave approximation by writing $x=\hat{x} / \epsilon, L_{1}=\hat{L}_{1} / \epsilon, L_{2}=\hat{L}_{2} / \epsilon$, $v=\epsilon \hat{v}, t=\hat{t} / \epsilon$ and $R=\hat{R} / \epsilon$, assuming that $\hat{R}, \hat{L}_{1}$ and $\hat{L}_{2}$ are all $O$ (1) quantities. Dropping the hats, (2.1) reduces with error $O\left(\epsilon^{2}\right)$ to the unsteady boundary-layer equations,

$u_{x}+v_{y}=0$,

$u_{t}+u u_{x}+v u_{y}=-p_{x}+R^{-1} u_{y y}$,

$p_{y}=0$.

Along the rigid parts of the channel walls we impose $(u, v)=$ $(0,0)$, while along $y=h(x, t), 0<x<1$ the no-slip and kinematic conditions are

$u=0, \quad h_{t}=v$.

The membrane shape is determined by the normal stress balance, which reduces, with error $O\left(\epsilon^{2}\right)$, to

$p=p_{e}(x)+d h_{t}-T h_{x x} \quad(0<x<1, y=h)$,

where $p_{e}(x)$ is given by (2.3). We assume that the tension parameter $T=\epsilon^{2} T_{0} /\left(\rho U_{0}^{2} a\right)$ and the damping parameter $d=\epsilon d_{0} /\left(\rho U_{0}\right)$ are $O(1)$. Variations in tension due to viscous stresses are then $O\left(\epsilon^{2}\right)$ and can be neglected. We also require that $p\left(-L_{1}, t\right)=p_{u}$ and $p\left(1+L_{2}, t\right)=0$.

\subsection{Integral approximation} flux

Integrating (2.4) across the width of the channel, the volume $q(x, t)=\int_{0}^{h} u \mathrm{~d} y$

satisfies the mass and momentum integral equations

$h_{t}+q_{x}=0$,

$q_{t}+\left(\int_{0}^{h} u^{2} \mathrm{~d} y\right)_{x}=-p_{x} h+R^{-1}\left[u_{y}\right]_{0}^{h}$.

To close this system of equations we employ a von KarmanPolhausen approximation, and assume that the velocity profile is everywhere parabolic. Setting $u=6 q y(h-y) / h^{3}$, (2.7) becomes

$h_{t}+q_{x}=0$,

$q_{t}+\frac{6}{5}\left(\frac{q^{2}}{h}\right)_{x}=-h p_{x}-\frac{12 q}{R h^{2}}$.

Where the channel has rigid walls $(h=1), q=q(t)$ and

$q_{t}=-p_{x}-\frac{12 q}{R}$.

The model therefore captures in a simple way the unsteady inertial and viscous resistance of the fluid in each rigid segment (the convective inertial resistance vanishes due to the assumption of uniform Poiseuille flow in the rigid segments). With pressures prescribed at $x=-L_{1}$ and $x=1+L_{2},(2.9)$ may be solved to give the pressures at the upstream and downstream ends of the compliant segment,

$p=p_{u}-\left(\frac{12 q}{R}+q_{t}\right) L_{1} \quad(x=0)$,

$p=\left(\frac{12 q}{R}+q_{t}\right) L_{2} \quad(x=1)$.

Combining (2.3), (2.6), (2.8) and (2.10), the simplified model for flow in the compliant segment of the channel reduces to

$$
\begin{aligned}
h_{t}+q_{x}=0, & \\
q_{t}+\frac{6}{5}\left(\frac{q^{2}}{h}\right)_{x}= & T h h_{x x x}-d h h_{x t} \\
& +\frac{12}{R}\left(h-\frac{q}{h^{2}}\right) \quad(0 \leqslant x \leqslant 1),
\end{aligned}
$$

with boundary conditions

$h=1, \quad T h_{x x}=L_{1}\left[\frac{12}{R}(q-1)+q_{t}\right] \quad(x=0)$,

$h=1, \quad T h_{x x}=-L_{2}\left[\frac{12}{R}(q-1)+q_{t}\right] \quad(x=1)$.

Solutions of (2.11) are parameterised by the modified Reynolds number $R=\epsilon \rho U_{0} a / \mu$, the tension parameter $T$, the damping parameter $d$ and the lengths $L_{1}$ and $L_{2}$ of the upstream and downstream rigid segments of the channel. In this paper, non-zero wall damping is employed only to induce an irreversible energy transfer from the fluid to the wall when classifying local instabilities [32-34]. The PDEs (2.11a) (with $d=0$ ) are essentially the same as those derived by Shkadov $[35,36]$, and subsequently studied by, for example, Trifonov and Tsvelodub [37,38], to describe instabilities of thin falling films in unbounded domains. More sophisticated approaches to the derivation of evolution equations for such systems are discussed by Scheid et al. [39]. Bessems et al. [40] have suggested an integral formulation that may be more suitable for high-frequency motions which permits a phase difference between the flow and the wall shear stress. Thin film instabilities governed by analogues of (2.11a) have been shown to be convectively, rather than absolutely, unstable (Brevdo et al. [41]).

Guneratne and Pedley [42] have demonstrated the existence of static eigenmodes of essentially the physical system shown in Fig. 1 using rational high-Reynolds-number asymptotics. We will also consider the static version of (2.11), for which $q$ is constant and

$-\frac{6 q^{2}}{5} \frac{h_{x}}{h^{2}}=T h h_{x x x}+\frac{12}{R}\left(h-\frac{q}{h^{2}}\right) \quad(0 \leqslant x \leqslant 1)$,

subject to

$h=1, \quad T h_{x x}=\frac{12}{R} L_{1}(q-1) \quad(x=0)$,

$h=1, \quad T h_{x x}=-\frac{12}{R} L_{2}(q-1) \quad(x=1)$.

This is a third-order system with four boundary conditions, so we expect $q$ to be an eigenvalue and $h$ the corresponding eigensolution, both parameterised by $T, R, L_{1}$ and $L_{2}$.

Solutions to the non-linear static problem (2.12) were computed by discretising the governing equations using finite differences and solving the resulting system with Newton's method. To illustrate the stability properties of the uniform and collapsed states, we also present below direct simulations of the full unsteady, non-linear problem (2.11) using a first-order implicit time-stepping scheme, 
where spatial derivatives were discretised using fourth-order finite differences. We found that an implicit representation of the highest spatial derivative in each governing equation (and an explicit representation of the remaining terms) was sufficient to ensure numerical stability. We found that the accuracy of the scheme was increased (specifically in the estimation of the critical Reynolds number for both mode- 1 and mode- 2 oscillations, as compared to the linear theory, discussed below) by using a fully-implicit representation of the time derivatives in the boundary conditions.

\subsection{Energy balance}

In Section 5 we will describe the energy budgets of various modes of oscillation. The kinetic energy of the flow in the collapsible segment of the channel (scaled on $\rho U_{0}^{2} a$ ) takes the form

$K(x, t)=\int_{0}^{h} \frac{1}{2} u^{2} \mathrm{~d} y=\frac{3}{5} \frac{q^{2}}{h}$,

again assuming a parabolic velocity profile. By multiplying both sides of the momentum equation in (2.11a) by $6 q / 5 h$ and integrating across the channel width, we derive the corresponding energy equation for flow in the collapsible segment, which takes the form

$\frac{3}{5}\left(\frac{q^{2}}{h}\right)_{t}+\frac{18}{25}\left(\frac{q^{3}}{h^{2}}\right)_{x}+\frac{3}{25}\left(\frac{q^{2} q_{x}}{h^{2}}\right)=-\frac{6}{5} q p_{x}-\frac{6}{5} \frac{12}{R} \frac{q^{2}}{h^{3}}$.

The first term on the left-hand side of (2.14) represents the rate of change of kinetic energy across the channel; the second represents the flux of kinetic energy; the third is a source of energy associated with the flow profile assumption (the approximate system is nonconservative). The first term on the right-hand side represents the pressure doing work (both at the ends of the channel and across the membrane) while the second represents the viscous dissipation along the channel.

In a similar manner we derive expressions for the energy transport in the rigid sections of the channel, which resembles (2.14) with $h=1$ and $q=q(t)$.

Integrating along the channel, we can sum the contributions from each region and split the energy equation into five components. We denote $\mathcal{K}$ as the net rate of change of kinetic energy, $\mathcal{F}$ as the net kinetic energy flux into the system, $\mathcal{P}$ as the rate of working of pressure forces upstream (no work is done downstream as $\left.p\left(1+L_{2}, t\right)=0\right), \mathcal{E}$ as the rate of working of pressure forces across the membrane and $\mathcal{D}$ as the rate of energy loss due to viscous dissipation. The energy source term due to the flow profile assumption is absorbed into the viscous dissipation and the energy equation in the limit of zero wall damping is expressed as

$\mathcal{K}+\mathcal{E}=\mathcal{F}+\mathcal{P}-\mathcal{D}$

where

$\mathcal{K}=\frac{3}{5} \frac{\partial}{\partial t}\left(\int_{0}^{1} \frac{q^{2}}{h} \mathrm{~d} x+\int_{-L_{1}}^{0} q^{2} \mathrm{~d} x+\int_{1}^{1+L_{2}} q^{2} \mathrm{~d} x\right)$

$\mathcal{F}=-\frac{18}{25}\left[\frac{q^{3}}{h^{2}}\right]_{x=0}^{x=1}$,

$\mathcal{P}=\left.\frac{6}{5} p_{u} q\right|_{x=0}$

$\mathcal{E}=\frac{6}{5} \int_{0}^{1} h_{t} p \mathrm{~d} x$

$$
\begin{aligned}
\mathcal{D}= & \frac{72}{5 R}\left(\int_{0}^{1} \frac{q^{2}}{h^{3}} \mathrm{~d} x+\int_{-L_{1}}^{0} q^{2} \mathrm{~d} x+\int_{1}^{1+L_{2}} q^{2} \mathrm{~d} x\right) \\
& +\frac{3}{25} \int_{0}^{1} \frac{q^{2} q_{x}}{h^{2}} \mathrm{~d} x .
\end{aligned}
$$

All the quantities in (2.16) scale in an identical manner in the long-wavelength approximation. Furthermore, we assume that the energy lost due to viscous dissipation does not lead to appreciable changes in temperature that might influence material parameters.

\section{Linear stability of uniform-width flow}

We now analyse the linear stability of the uniform state $h=1$, $q=1$, assuming the wall is free from damping $(d=0)$. In this section we seek global modes satisfying all the boundary conditions.

\subsection{Static solutions}

We begin by computing the static eigenmodes governed by (2.12). Linearising about the uniform width state, with $h=1+$ $\epsilon_{1} H(x), q=1+\epsilon_{1} Q$ (where $H$ and $Q$ are real and $\epsilon_{1} \ll 1$ ), assuming that $Q \neq 0, \hat{H}(x)=H(x) / Q$ satisfies the following inhomogeneous problem:

$$
\begin{aligned}
& T \hat{H}_{x x x}+\frac{6}{5} \hat{H}_{x}+\frac{36}{R} \hat{H}=\frac{12}{R}, \\
& \hat{H}=0, \quad T \hat{H}_{x x}=(12 / R) L_{1} \quad(x=0), \\
& \hat{H}=0, \quad T \hat{H}_{x x}=-(12 / R) L_{2} \quad(x=1) .
\end{aligned}
$$

The problem can be simplified further by setting $r=12 /(R T), \tau=$ $6 /(5 T), \lambda^{3}=3 r$ and $\tilde{H}=\hat{H} / r$, giving

$\tilde{H}_{x x x}+\tau \tilde{H}_{x}+\lambda^{3} \tilde{H}=1$,

$\tilde{H}=0 \quad \tilde{H}_{x x}=L_{1}, \quad(x=0)$,

$\tilde{H}=0, \quad \tilde{H}_{x x}=-L_{2} \quad(x=1)$

By writing $\tilde{H}=\lambda^{-3}+\operatorname{Re}\left(\sum_{i=1}^{3} A_{i} \mathrm{e}^{m_{i} x}\right)$, where the $m_{i}$ are the roots of

$m^{3}+\tau m+\lambda^{3}=0$

and the $A_{i}$ are constants, solutions satisfying the boundary conditions (3.2b)-(3.2c) exist provided

$\left|\begin{array}{cccc}\lambda^{-3} & 1 & 1 & 1 \\ -L_{1} & m_{1}^{2} & m_{2}^{2} & m_{3}^{2} \\ \lambda^{-3} & \mathrm{e}^{m_{1}} & \mathrm{e}^{m_{2}} & \mathrm{e}^{m_{3}} \\ L_{2} & m_{1}^{2} \mathrm{e}^{m_{1}} & m_{2}^{2} \mathrm{e}^{m_{2}} & m_{3}^{2} \mathrm{e}^{m_{3}}\end{array}\right|=0$.

Solving for $\lambda$ yields neutral stability curves for static modes in $(T, R)$-space that provide a useful independent test of solutions of the full dynamic problem. Some representative results are presented in Fig. 2, where we classify a mode- $n$ disturbance as one for which $|H|$ has $n$ extrema for $x \in(0,1)$.

\subsection{Oscillatory solutions}

Returning to the unsteady problem (2.11), setting

$h=1+\operatorname{Re}\left(H(x) \mathrm{e}^{\sigma t}\right), \quad q=1+\operatorname{Re}\left(Q(x) \mathrm{e}^{\sigma t}\right)$,

where $H$ and $Q$ are complex, $|H| \ll 1,|Q| \ll 1$ and $\sigma$ is a complex growth rate, we obtain the following eigenvalue problem: 


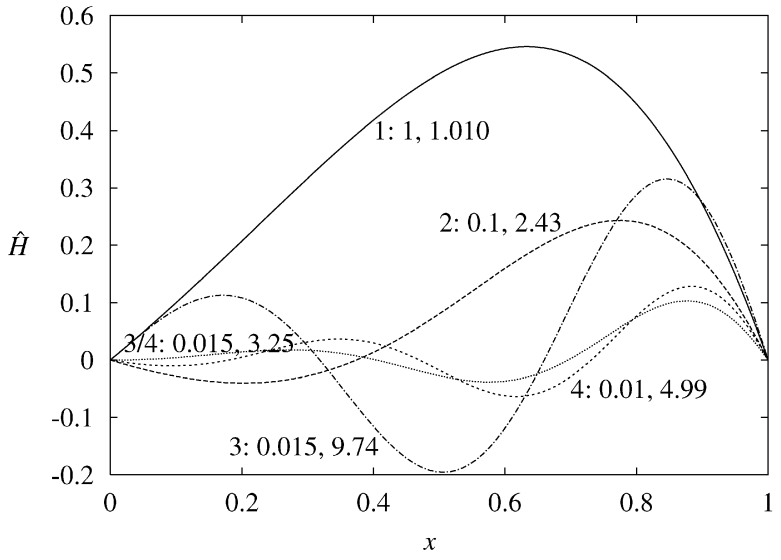

Fig. 2. Static eigenmodes, showing the amplitude of the membrane displacement. Labels show $n: T, R$, where an mode- $n$ disturbance is one for which $|H|$ has $n$ humps. The curve labelled $3 / 4$ is an eigenmode at the transition between a mode- 3 oscillation and a mode- 4 oscillation.

$$
\begin{aligned}
& T Q^{\prime \prime \prime \prime}+\frac{6}{5} Q^{\prime \prime}+\left(\frac{36}{R}+\frac{12 \sigma}{5}\right) Q^{\prime}+\left(\frac{12 \sigma}{R}+\sigma^{2}\right) Q=0 \\
& \quad(0<x<1), \\
& Q^{\prime}(0)=Q^{\prime}(1)=0, \\
& 0=T Q^{\prime \prime \prime}(0)+L_{1} \sigma\left(\frac{12}{R}+\sigma\right) Q(0), \\
& 0=T Q^{\prime \prime \prime}(1)-L_{2} \sigma\left(\frac{12}{R}+\sigma\right) Q(1),
\end{aligned}
$$

where the primes denote a derivative with respect to $x$.

Letting $k_{j}$ be the four roots of

$T k^{4}-\frac{6}{5} k^{2}+\left(\frac{36}{R}+\frac{12 \sigma}{5}\right) i k+\left(\frac{12 \sigma}{R}+\sigma^{2}\right)=0$,

we can write the solution of (3.6a) as $Q=\sum_{j=1}^{4} A_{j} \mathrm{e}^{i k_{j} x}$ (assuming the roots are distinct), for some constants $A_{j}$. The boundary conditions (3.6b), (3.6c), (3.6d) then demand that $\operatorname{det} M=0$, where the matrix $M_{r s}$ has, for $s=1,2,3,4$,

$M_{1 s}=i k_{s}$,

$M_{2 s}=i k_{s} \mathrm{e}^{i k_{s}}$,

$M_{3 s}=-i T k_{s}^{3}+L_{1} \sigma(\sigma+12 / R)$,

$M_{4 s}=-i T k_{s}^{3} \mathrm{e}^{i k_{s}}-L_{2} \sigma(\sigma+12 / R) \mathrm{e}^{i k_{s}}$.

We can then find $\sigma\left(R, T, L_{1}, L_{2}\right)$ numerically and seek parameter values where $\operatorname{Re}(\sigma)=0$, which correspond to purely oscillatory solutions.

\subsection{Overview of parameter space}

The effect of varying the lengths of the upstream and downstream rigid segments on a mode- 1 oscillatory instability was investigated by Jensen and Heil [19]. They found that instability of this global mode was promoted by having the length $L_{2}$ much greater than $L_{1}$. Throughout this paper we therefore set $L_{1}=1$ and $L_{2}=10$ and consider behaviour across $(T, R)$ parameter space.

Fig. 3(a, b) shows loci in $(T, R)$ parameter space for which an eigenvalue has zero real part. There are multiple eigenmodes that lose stability as parameters are varied. Fig. 3(a) maps out the static and oscillatory neutral curves; shaded regions in Fig. 3(b) indicate where the uniform state is unstable to either a static or oscillatory instability.

Mode 1, for which $|H|$ has a single hump, becomes unstable via a Hopf bifurcation as $R$ increases across the neutral curve
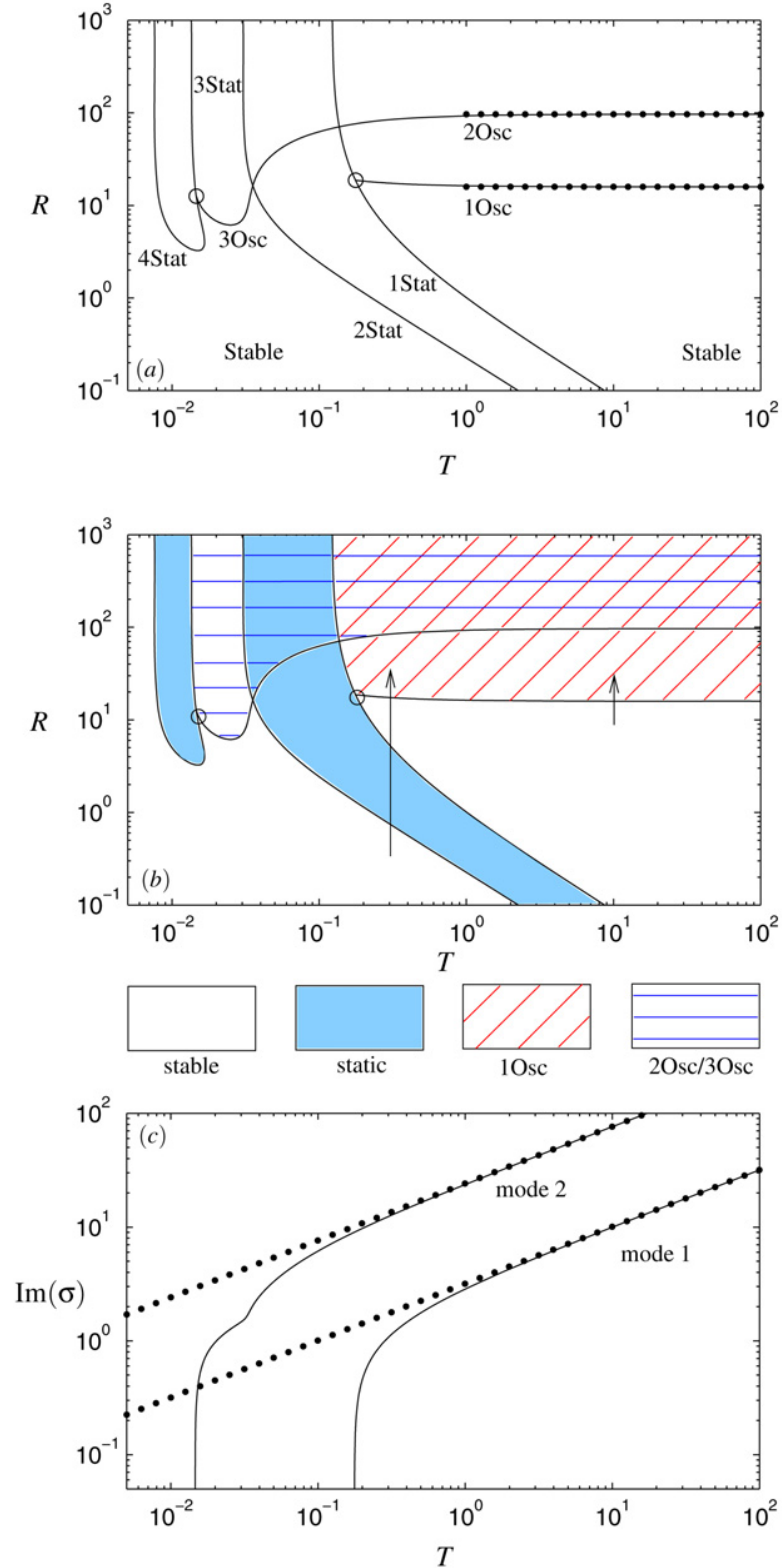

Fig. 3. (a) Stability boundaries in $(T, R)$-space for $L_{1}=1, L_{2}=10$. nOsc shows boundaries across which the uniform state becomes unstable to mode $n$ via a Hopf bifurcation. $n$ Stat shows boundaries across which it loses stability to mode $n$ via a transcritical bifurcation. Takens-Bogdanov points are marked with open circles. Horizontal asymptotes $R_{1}=15.86$ and $R_{2}=96.72$ of 10 sc and 20sc are shown (filled black circles) for $T \geqslant 1$. (b) An additional view of stability boundaries in $(T, R)$ space, using shading to distinguish the regions where the uniform base state is unstable to static or oscillatory modes. (c) Frequencies of modes 1 and 2, showing respective asymptotes $3.1807 T^{1 / 2}$ and $24.1251 T^{1 / 2}$ as $T \rightarrow \infty$ (see Section 3.4). Arrows in (b) show paths mapped out in Figs. 10 and 11 below.

marked '10sc.' The corresponding eigenvalue has non-zero imaginary part, representing the frequency of the neutrally stable oscillation (the lower curve in Fig. 3(c)). The neutral curve asymptotes to $R=R_{1}$, say, where $R_{1} \approx 16$, and the frequency increases proportionally to $T^{1 / 2}$, as $T \rightarrow \infty$ (Fig. 3(c)). Mode 1 also becomes unstable via a transcritical bifurcation (with a purely real eigenvalue passing through zero) as $T$ decreases across the line marked '1Stat' in Fig. 3(a). The static and oscillatory mode-1 curves intersect at $T \approx 0.177, R \approx 18.77$. The uniform state is linearly stable for $(T, R)$ beneath (and to the right of) these two neutral curves (Fig. 3(b)). The frequency of the oscillatory mode falls to zero close to the intersection point with the static neutral curve (Fig. 3(c)). 

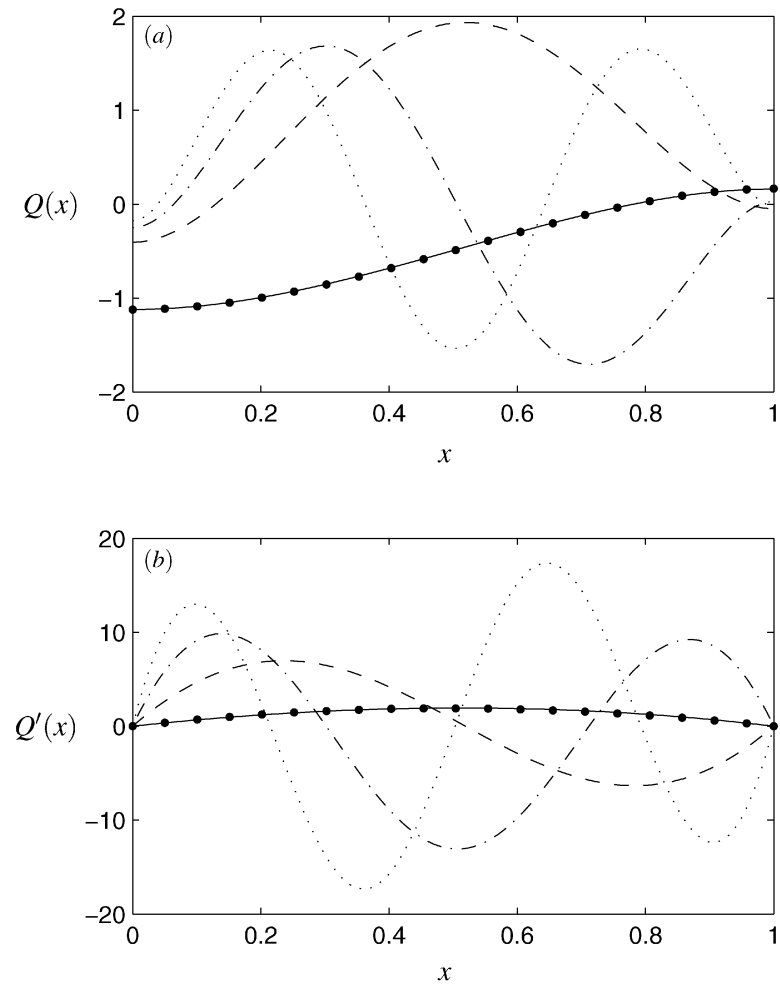

Fig. 4. (a) $Q_{0}(x)$ and (b) $Q_{0}^{\prime}(x)$ (proportional to membrane displacement) for modes $1-4$, with $L_{1}=1$ and $L_{2}=10$. Mode $n$ has $n$ extrema in $Q_{0}^{\prime}(x)$. The filled black circles correspond to the eigenfunction found by direct numerical simulation (appropriately scaled) of the full non-linear problem for $R=15.9, T=10$. Here $L_{1}=1$ and $L_{2}=10$.

The interaction of a Hopf and a transcritical bifurcation suggests that near the co-dimension-2 point the system will exhibit a global (Takens-Bogdanov) bifurcation (Guckenheimer and Holmes [43]).

Fig. 3(a) also shows oscillatory and static neutral curves for mode 2 . For $T \rightarrow \infty$, the oscillatory neutral curve '20sc' asymptotes to $R=R_{2}$, where $R_{2} \approx 97$, and the frequency again increases like $T^{1 / 2}$ (Fig. 3(c)). The uniform state is stable for low $T$ and $R$ (bottom left of Fig. 3(a, b)), losing stability to a mode-2 static solution (again via a transcritical bifurcation) as $T$ increases across the neutral curve marked ' 2 Stat'. The resulting mode-2 static solution has the membrane inflated at the upstream end of the channel and collapsed downstream. We show in Fig. 11(a) below how this branch of static solutions connects to the branch of mode-1 static solutions emerging from the nearby 1Stat curve. Although the mode-2 oscillatory and static neutral curves intersect (near $T=0.0354, R=16.32$ ), the eigenvalues do not coalesce at this intersection point because the oscillatory eigenfunction instead develops mode- 3 characteristics. For lower $T$, what is now a mode- 3 oscillatory neutral curve (30sc) coalesces with a mode- 3 static neutral curve (3Stat) at $T \approx 0.015, R \approx 12.5$, a second TakensBogdanov point. The mode-3 static neutral curve (3Stat) is itself joined to a mode- 4 static neutral curve (4Stat, Fig. 3(a, b)). In contrast, the mode- 1 and 2 static neutral curves remain distinct, and extend down to $R \rightarrow 0$ (Fig. 3(a, b)). Determining further solution branches was difficult numerically, and not thought to be necessary given that the primary oscillatory instability of the uniform state is always to mode-1. Nevertheless, these structures give an indication of the dynamical richness of this system.

Typical static eigenfunctions of modes 1 to 4 are shown in Fig. 2, and high-frequency oscillatory eigenfunctions (determined using large-tension asymptotics below) are shown in Fig. 4 below. A consistent trend for $L_{1}=1$ and $L_{2}=10$ is that static membrane deformations have larger amplitudes towards the downstream end of the collapsible segment (Fig. 2), while oscillatory modes have larger fluxes at the upstream end (Fig. 4).

We now briefly describe the asymptotic structure of the dynamic neutral curves for large $T$. In this limit we expect the integral approximation of Section 2.2 to be inaccurate, and indeed Jensen and Heil [19] have considered this limit in detail using formal asymptotics of the two full two-dimensional system (2.4)(2.6), finding a different scaling behaviour in the neutral curves. Nevertheless the simplified model captures much of the significant physics and merits investigation.

\subsection{Large-tension asymptotics for oscillatory modes}

The results shown in Fig. 3 suggest that a simplified structure exists in the limit $T \gg 1$ for the oscillatory modes described in Section 3.2. We investigate this by setting $\sigma=T^{1 / 2} \hat{\sigma}$, and expand with

$Q=Q_{0}(x)+T^{-1 / 2} Q_{1}(x)+\cdots, \quad \hat{\sigma}=\hat{\sigma}_{0}+T^{-1 / 2} \hat{\sigma}_{1}+\cdots$

At leading order, (3.6) becomes

$Q_{0}^{\prime \prime \prime \prime}+\hat{\sigma}_{0}^{2} Q_{0}=0$,

$Q_{0}^{\prime}=0, \quad Q_{0}^{\prime \prime \prime}+L_{1} \hat{\sigma}_{0}^{2} Q_{0}=0 \quad(x=0)$,

$Q_{0}^{\prime}=0, \quad Q_{0}^{\prime \prime \prime}-L_{2} \hat{\sigma}_{0}^{2} Q_{0}=0 \quad(x=1)$.

This is an eigenvalue problem, for which $\hat{\sigma}_{0}= \pm i \beta_{n}, n=1,2, \ldots$. Writing $z=\sqrt{\beta_{n}}$, the eigenfunction is of the form

$Q_{0}=A_{1} \cosh (z x)+A_{2} \sinh (z x)+A_{3} \cos (z x)+A_{4} \sin (z x)$,

where the $z$ are the roots of

$\left|\begin{array}{cccc}0 & 1 & 0 & 1 \\ L_{1} z & -1 & L_{1} z & 1 \\ \sinh z & \cosh z & -\sin z & \cos z \\ \sinh z+L_{2} z \cosh z & \cosh z+L_{2} z \sinh z & \sin z+L_{2} z \cos z & -\cos z+L_{2} z \sin z\end{array}\right|=0$.

The four lowest modes are shown in Fig. 4, computed assuming $A_{4}=1$. In all cases, with $L_{1}=1$ and $L_{2}=10,\left|Q_{0}(0)\right|>\left|Q_{0}(1)\right|$. The frequencies obtained by solving (3.12) agree closely with solutions of the full problem, as shown by the asymptotes in Fig. 3(c) for $T>5$ : for our choice of $L_{1}$ and $L_{2}, \beta_{1} \approx 3.1807$ and $\beta_{2} \approx$ 24.1251. These eigenmodes are high-frequency normal modes of the system, arising through a balance between the inertia of the fluid in the entire length of the channel and the restoring force of membrane tension. Neither the mean flow nor viscous effects appear at leading order. The same eigenmodes were described in Jensen and Heil [19], who showed how the frequencies $\beta_{n}$ depend on $L_{1}$ and $L_{2}$.

We also follow Jensen and Heil [19] in using a solvability condition at the next order to find the growth-rate of the eigenmodes. For each mode, from (3.6),

$$
\begin{aligned}
& \mathcal{L}_{n} Q_{1} \equiv Q_{1}^{\prime \prime \prime \prime}-\beta_{n}^{2} Q_{1}=i \beta_{n}\left[-\frac{12}{5} Q_{0}^{\prime}-\left(\frac{12}{R}+2 \hat{\sigma}_{1}\right) Q_{0}\right] \\
& Q_{1}^{\prime}=0, \quad Q_{1}^{\prime \prime \prime}-L_{1} \beta_{n}^{2} Q_{1}=-i \beta_{n} L_{1} Q_{0}\left(\frac{12}{R}+2 \hat{\sigma}_{1}\right) \\
& \quad(x=0), \\
& Q_{1}^{\prime}=0, \quad Q_{1}^{\prime \prime \prime}+L_{2} \beta_{n}^{2} Q_{1}=i \beta_{n} L_{2} Q_{0}\left(\frac{12}{R}+2 \hat{\sigma}_{1}\right) \\
& (x=1) .
\end{aligned}
$$

Under the inner product

$$
\langle f, g\rangle=\int_{0}^{1} f g \mathrm{~d} x
$$


for functions $f(x), g(x)$, the operator $\mathcal{L}_{n}$ subject to the homogeneous boundary conditions in (3.10) is self-adjoint. Considering $\left\langle Q_{0}, \mathcal{L}_{n} Q_{1}\right\rangle$, integration by parts leads to

$$
\begin{aligned}
& \left(\frac{12}{R}+2 \hat{\sigma}_{1}\right)\left[L_{1} Q_{0}^{2}(0)+L_{2} Q_{0}^{2}(1)+\left\langle Q_{0}, Q_{0}\right\rangle\right] \\
& \quad=-\frac{12}{5}\left\langle Q_{0}, Q_{0}^{\prime}\right\rangle .
\end{aligned}
$$

This relationship captures the balance at this order between viscous resistance in the entire channel (the term $12 / R$ ), unsteady inertia in the entire channel (the term $2 \hat{\sigma}_{1}$ ) and convective inertia in the collapsible segment (the term $-\left\langle Q_{0}, Q_{0}^{\prime}\right\rangle=\frac{1}{2}\left[Q_{0}^{2}(0)-Q_{0}^{2}(1)\right]$ ). It follows that these modes are neutrally stable for $R=R_{n}$ where

$R_{n}=\frac{10\left[L_{1} Q_{0}^{2}(0)+L_{2} Q_{0}^{2}(1)+\left\langle Q_{0}, Q_{0}\right\rangle\right]}{Q_{0}^{2}(0)-Q_{0}^{2}(1)}$,

and $\hat{\sigma}_{1}<0$ for $R<R_{n}$. Thus for our choice of $L_{1}$ and $L_{2}$, for which $Q_{0}^{2}(0)-Q_{0}^{2}(1)>0$, using $Q_{0}(x)$ such as shown in Fig. $4, R_{1} \approx$ 15.86 and $R_{2} \approx 96.72$. These asymptotes are shown in Fig. 3(a); there is close agreement for $T>5$. Note also that the terms $6 Q^{\prime \prime} / 5$ and $36 Q^{\prime} / R$ in (3.6), representing Bernoulli effects and the pressure gradient imposed by the external pressure distribution, play no role at this order in determining the stability threshold.

The expression (3.16) resembles the critical Reynolds number derived by Jensen and Heil [19], which they denote as $r_{c 0}$. Their paper contains a discussion of how this critical Reynolds number (and the corresponding oscillatory frequency) varies with the lengths of the upstream and downstream rigid sections.

In Appendix A we extend this analysis to account for weakly non-linear effects, demonstrating that mode-1 oscillations in the large-tension limit bifurcate subcritically from the uniform state as $R$ increases through $R_{1}$. The mechanism allowing energy transfer from the mean flow is examined in Section 5 below.

\section{Local vs. global stability}

Having shown the existence of several modes of self-excited oscillation in the global system (Fig. 3(a)), and how a high-frequency normal mode of the system can be destabilised by the mean flow (Section 3.4), we now consider the underlying local (unbounded) problem, to classify the waves that contribute to the primary oscillatory instability (Section 4.1) and to demonstrate the essential role of boundary conditions (Section 4.2 ) in sustaining mode-1 oscillations. Setting $q=1+\hat{q}, h=1+\hat{h}$ in (2.11) and assuming $\hat{q}, \hat{h} \ll 1$, we express the linearised governing equations in operator form as

$$
\begin{aligned}
\mathcal{D}_{1}\left[\begin{array}{l}
\hat{h} \\
\hat{q}
\end{array}\right] & \equiv\left[\begin{array}{cc}
\frac{\partial}{\partial t} & \frac{\partial}{\partial x} \\
-T \frac{\partial^{3}}{\partial x^{3}}+d \frac{\partial^{2}}{\partial x \partial t}-\frac{6}{5} \frac{\partial}{\partial x}-\frac{36}{R} & \frac{\partial}{\partial t}+\frac{12}{5} \frac{\partial}{\partial x}+\frac{12}{R}
\end{array}\right]\left[\begin{array}{l}
\hat{h} \\
\hat{q}
\end{array}\right] \\
& =0 .
\end{aligned}
$$

It is convenient to retain the wall-damping term in this section, as this will enable us to classify the waves in the system (Section 4.2; Appendix B).

\subsection{Characterising the local modes}

To establish criteria capable of discriminating between stable, convectively unstable and absolutely unstable flows (Huerre [30]; Huerre and Rossi [31]), we force the governing equation according to

$\mathcal{D}_{1}\left[\begin{array}{l}\hat{h} \\ \hat{q}\end{array}\right]=\left[\begin{array}{c}0 \\ \delta(x) \delta(t)\end{array}\right]$.

Fourier transforming (4.2) in space and time according to

$\tilde{\tilde{f}}(k, \omega)=\int_{-\infty}^{\infty} \int_{-\infty}^{\infty} \mathrm{e}^{-i(k x-\omega t)} f(x, t) \mathrm{d} x \mathrm{~d} t$,

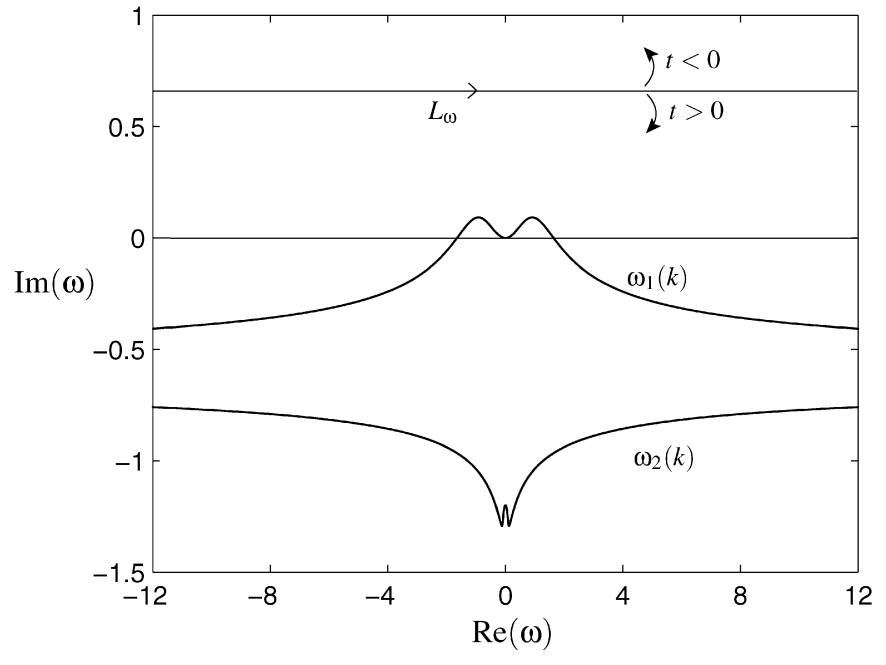

Fig. 5. The temporal branches (zeros of $D(k, \omega)$ for $k$ varying along the real axis). Here $T=10, R=10, L_{1}=1, L_{2}=10$ and $d=0$. Also included is the initial position of the contour $L_{\omega}$, which is closed from above for $t<0$ and from below for $t>0$ to satisfy causality.

where $k$ (wavenumber) and $\omega$ (frequency) are arguments of the transformed variables, we obtain

$D(k, \omega) \tilde{\tilde{G}}(k, \omega)=1$,

where

$D(k, \omega) \equiv \omega^{2}+\frac{6}{5}\left(k^{2}-2 \omega k\right)-T k^{4}+i d \omega k^{2}-\frac{12 i}{R}(3 k-\omega)$

is the dispersion relation, which is zero for normal modes. When expressed in less conventional notation, (4.5) is equivalent to (3.7) with $\sigma=-i \omega$ and $d=0 . G$ denotes the Green's function response to point forcing (where $\tilde{\tilde{G}}=-i \tilde{\tilde{q}} / \omega=-i \tilde{\tilde{h}} / k$ ).

For $k$ real, zeros of $D(k, \omega)$ in the $\omega$-plane are known as temporal branches. In this case we have two temporal branches, $\omega_{1}$ and $\omega_{2}$, plotted in Fig. 5 for illustrative parameters. Similarly, for $\omega$ real, zeros of $D(k, \omega)$ in the $k$-plane are known as spatial branches, of which there are four (as the problem is fourth order in space). Generalised spatial branches (with $\omega$ complex) are illustrated in Fig. 6.

In general $D(k, \omega)=0$ defines two distinct frequencies $\omega$ for a given $k$. However, at four distinct points in the $k$-plane (identified by black dots in Fig. 6$)$ the function $\omega(k)$ becomes single-valued. Two of these branch points lie along the $\operatorname{Im}(k)$ axis in separate halves of the $k$-plane, whilst the other two have the same imaginary part but real parts of opposite sign. We therefore define two branch cuts: the first between the two branch points along the $\operatorname{Im}(k)$ axis such that the cut passes through the origin; the second along a line of constant imaginary part, joining the remaining two branch points at infinity. These are illustrated in Fig. 6 .

Returning to Fig. 5, we see evidence of local instability $(\operatorname{Im}(\omega)>$ 0 ) for a small band of wavenumbers centred around the origin (where $k=0$ ). To verify this formally, we construct a regular perturbation in $k \ll 1$, with $D=0$ giving

$\omega=3 k+\frac{i R}{4} k^{2}+O\left(k^{3}\right)$.

Thus $\operatorname{Im}(\omega)>0$ for all $k \ll 1$ and the system is locally unstable for all $(T, R, d)$.

To classify this instability we return to the forced system (4.4) and invert the Fourier transforms by integrating along contours $L_{\omega}$ in the $\omega$-plane and $F_{k}$ in the $k$-plane, which implies

$G(x, t)=\frac{1}{(2 \pi)^{2}} \int_{F_{k}} \int_{L_{\omega}} \frac{\mathrm{e}^{i(k x-\omega t)}}{D(k, \omega)} \mathrm{d} \omega \mathrm{d} k$. 

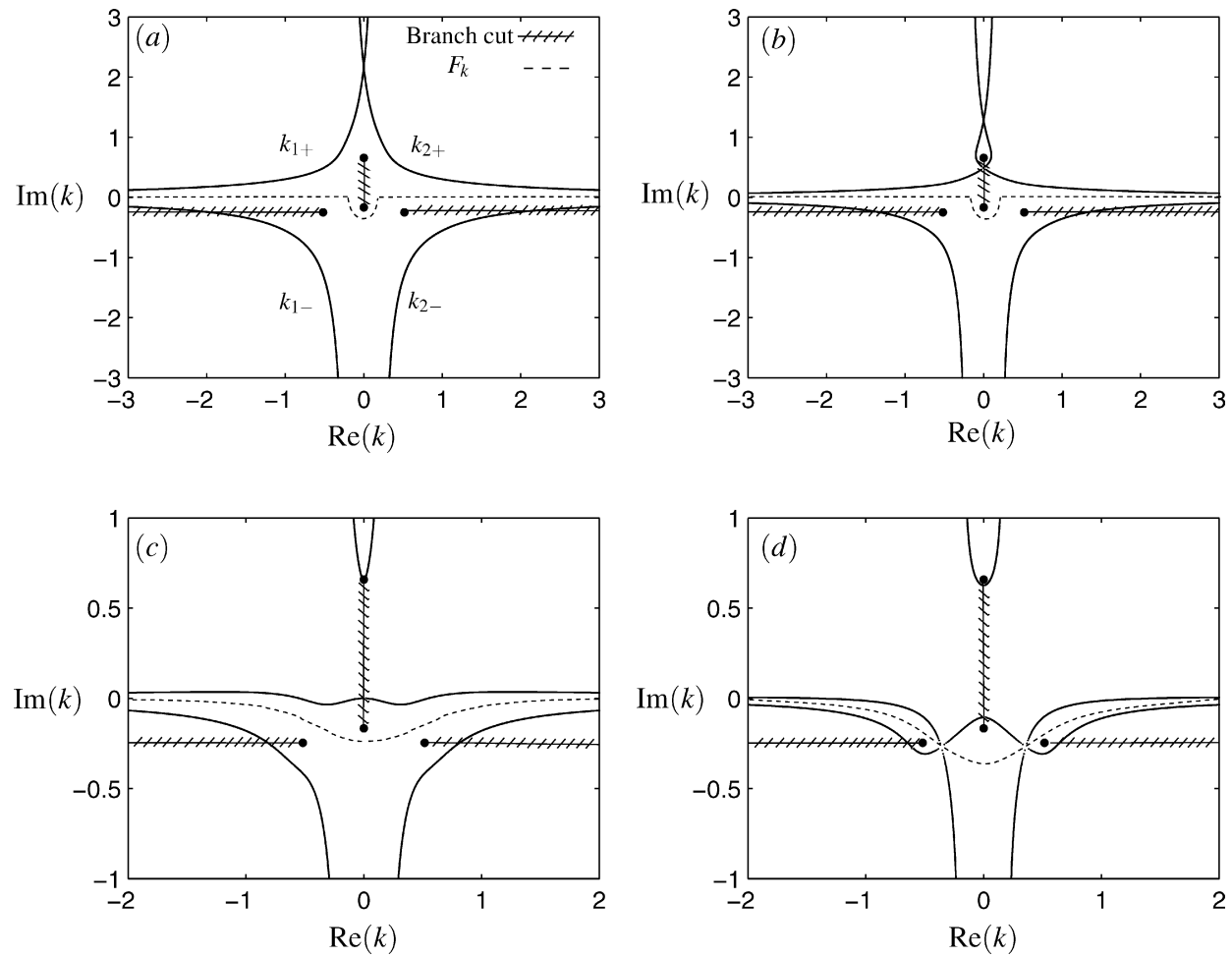

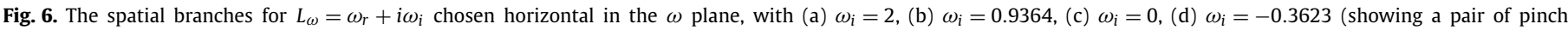
points, those with the greatest imaginary part). The filled black circles are the branch points and checked lines denote branch cuts. Here $T=10, R=10$ and $d=0$.

Causality demands that we initially place the contour $L_{\omega}$ as a horizontal line above the (generalised) temporal branches (Fig. 5), from which we can calculate the corresponding four generalised spatial branches, two in each half of the $k$-plane (Fig. 6(a)). Causality also demands that the contour $F_{k}$ separate the generalised spatial branches that originate in separate halves of the $k$-plane, and so we initially choose $F_{k}$ along the $\operatorname{Re}(k)$ axis, with an appropriate deformation below the branch cut at the origin.

We can legitimately deform $L_{\omega}$ downwards without changing the value of the integral as long as $F_{k}$ can be suitably deformed to ensure the partition between spatial branches originating in the upper and lower $k$-plane (Fig. $6(\mathrm{~b}-\mathrm{c})$ ). As $L_{\omega}$ is lowered, the generalised spatial branches from separate halves of the $k$-plane approach one another. The contour $L_{\omega}$ eventually touches the upper generalised temporal branch, where correspondingly $F_{k}$ becomes pinched between two generalised spatial branches (Fig. 6(d)). Further lowering of the contour $L_{\omega}$ is futile as causality has been violated. Pinching of the generalised spatial branches occurs at two points simultaneously, with their wavenumbers denoted $k_{01}$ and $k_{02}$ and frequencies denoted $\omega_{01}$ and $\omega_{02}$. These frequencies have identical imaginary part and are global maxima of $\operatorname{Im}\left(\omega_{j}\right)$ for all $j$.

Having identified these stationary points, we use the method of steepest descents to evaluate the Green's function as $t \rightarrow \infty$, which has the form

$$
\begin{aligned}
G(x, t) \sim & \frac{1}{2 \sqrt{2 \pi}}\left(\frac{\mathrm{e}^{\left(\frac{i \pi}{4}+i\left(k_{01} x-\omega_{01} t\right)\right)}}{\frac{\partial D}{\partial \omega}\left(k_{01}, \omega_{01}\right)}\left[t \frac{\partial^{2} \omega}{\partial k^{2}}\left(k_{01}\right)\right]^{-1 / 2}\right) \\
& +\frac{1}{2 \sqrt{2 \pi}}\left(\frac{\mathrm{e}^{\left(\frac{i \pi}{4}+i\left(k_{02} x-\omega_{02} t\right)\right)}}{\frac{\partial D}{\partial \omega}\left(k_{02}, \omega_{01}\right)}\left[t \frac{\partial^{2} \omega}{\partial k^{2}}\left(k_{02}\right)\right]^{-1 / 2}\right) .
\end{aligned}
$$

The growth rate of this Green's function in the stationary frame is given by $\operatorname{Im}\left(\omega_{01}\right)=\operatorname{Im}\left(\omega_{02}\right)$. Across $(T, R)$-parameter space for zero damping we find that this growth rate is negative, so despite the abundant global modes (Fig. 3(a)) the local modes appear always to be convectively (but not absolutely) unstable in the absence of wall damping.
In Appendix B we classify the modes in more detail using a framework based on activation energy (where local instabilities are classified as A, B or C [32-34]), and show that pinching involves a merging of an evanescent upstream-propagating Class A wave (static-divergence) and an unstable downstream-propagating Class B wave (travelling-wave flutter). These instabilities were first characterised in the study of boundary-layer flow past a compliant plate [32-34,46-48] and subsequently described in the study of plane Poiseuille flow in a compliant channel [5].

\subsection{Decomposition of a neutral mode: propagation and reflection}

Returning to the global stability problem (Section 3.2), the linearised wave profile $Q(x)$ (defined in (3.5b)) is a superposition of four waves. To illustrate, we consider $T=10, L_{1}=1, L_{2}=10$, where the critical Reynolds number for mode- 1 neutral stability is $R=15.91$ (Fig. 3(a)). The four corresponding wavenumbers are shown with crosses in the $k$-plane in Fig. 7 , where we also illustrate the trajectory of the wavenumber as $\operatorname{Im}(\omega)$ is decreased from some large value to zero while holding $\operatorname{Re}(\omega)$ fixed (equal to the real part of the neutrally stable mode). Two of these trajectories originate in the lower half $k$-plane (denoted $k_{1}$ and $k_{2}$ ) and define waves propagating upstream (using the classification described in Appendix B), while two originate in the upper half $k$-plane and define waves propagating downstream. Again following Appendix B, one each of the upstream- and downstream-propagating waves are stabilised by a small amount of wall damping and are thus Class B, so we classify these as travelling-wave flutter (denoted as TWF in Fig. 7). The remaining waves are destabilised by wall damping and are thus Class A (static-divergence). In the high-tension limit (see Section 3.4), the leading-order wavenumbers are either purely real (TWF) or purely imaginary (static-divergence), making the classification easier.

We now separate the contributions of wave propagation and wave reflection in generating a global instability, following Doaré and de Langre [45] and Gallaire and Chomaz [49]. We force the 


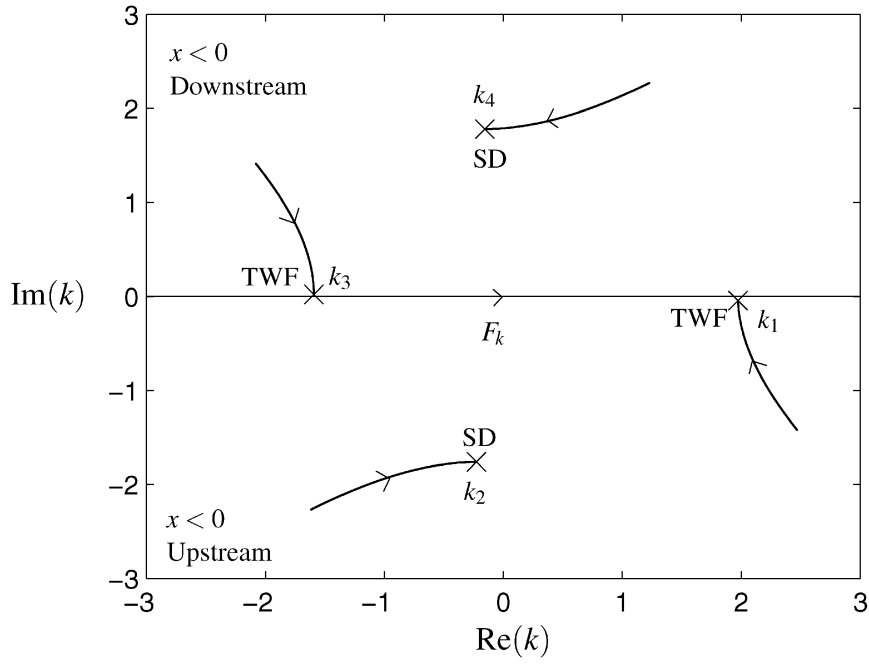

Fig. 7. The complex $k$-plane for a parameter combination on the mode- 1 neutral stability boundary. The solid lines illustrate the path of the wavenumber as the contour $L_{\omega}$ is lowered (in this case from $\operatorname{Im}(\omega)=20$ ) to 0 , holding $\operatorname{Re}(\omega)$ fixed (=-9.956). Here $T=10, R=15.9055, d=0, L_{1}=1$ and $L_{2}=10$.

system at fixed frequency $\omega_{f}$ at the downstream end of the collapsible segment, which generates two upstream waves with initial amplitudes $A_{1}$ and $A_{2}$ respectively. Their amplitudes at the upstream boundary, $A_{1}^{\prime}$ and $A_{2}^{\prime}$, are given by

$\left[A_{1}^{\prime}, A_{2}^{\prime}\right]^{T}=\mathbf{P}_{u}\left[A_{1}, A_{2}\right]^{T}$

where

$\mathbf{P}_{u}=\left[\begin{array}{cc}\mathrm{e}^{-i k_{1}} & 0 \\ 0 & \mathrm{e}^{-i k_{2}}\end{array}\right]$

is a matrix describing the contribution of upstream propagation. These waves are reflected at the upstream end of the collapsible segment, generating two downstream waves with wavenumbers $k_{3}$ and $k_{4}$ and initial amplitudes $A_{3}$ and $A_{4}$. We write the superposition of these waves for the flux in (3.5b) in the form

$Q(x)=A_{1}^{\prime} \mathrm{e}^{i k_{1} x}+A_{2}^{\prime} \mathrm{e}^{i k_{2} x}+A_{3} \mathrm{e}^{i k_{3} x}+A_{4} \mathrm{e}^{i k_{4} x}$.

Substituting (4.11) into the linearised upstream boundary conditions (3.6), we write these in the form

$\left[A_{3}, A_{4}\right]^{T}=\mathbf{R}_{u}\left[A_{1}^{\prime}, A_{2}^{\prime}\right]^{T}$,

where $\mathbf{R}_{u}$ denotes the contribution of the upstream reflection, which takes the form

$\mathbf{R}_{u}=\left[\begin{array}{ll}f\left(k_{1}, k_{4}, k_{3}\right) & f\left(k_{2}, k_{4}, k_{3}\right) \\ f\left(k_{1}, k_{3}, k_{4}\right) & f\left(k_{2}, k_{3}, k_{4}\right)\end{array}\right]$,

where

$f(a, b, c)=-\frac{T\left(i a b^{2}-i a^{3}\right)+C_{1}(1-a / b)}{T\left(i c b^{2}-i c^{3}\right)+C_{1}(1-c / b)}$

and $C_{1} \equiv-i L_{1} \omega_{f}\left(12 / R-i \omega_{f}\right)$.

These two waves propagate downstream, with their amplitudes $A_{3}^{\prime}$ and $A_{4}^{\prime}$ at the downstream end of the channel given by

$\left[A_{3}^{\prime}, A_{4}^{\prime}\right]^{T}=\mathbf{P}_{d}\left[A_{3}, A_{4}\right]^{T}$

where

$\mathbf{P}_{d}=\left[\begin{array}{cc}\mathrm{e}^{i k_{3}} & 0 \\ 0 & \mathrm{e}^{i k_{4}}\end{array}\right]$

is a matrix describing the contribution of downstream propagation. These two waves are reflected at the downstream end of the collapsible segment, generating two upstream waves, again with wavenumbers $k_{1}$ and $k_{2}$ and initial amplitudes $A_{1}^{\prime \prime}$ and $A_{2}^{\prime \prime}$. Once more we write the superposition of these waves in the form

$Q(x)=A_{1}^{\prime \prime} \mathrm{e}^{i k_{1} x}+A_{2}^{\prime \prime} \mathrm{e}^{i k_{2} x}+A_{3}^{\prime} \mathrm{e}^{i k_{3} x}+A_{4}^{\prime} \mathrm{e}^{i k_{4} x}$,

and upon substitution into the downstream boundary conditions (3.6c), we write these in the form

$\left[A_{1}^{\prime \prime}, A_{2}^{\prime \prime}\right]^{T}=\mathbf{R}_{d}\left[A_{3}^{\prime}, A_{4}^{\prime}\right]^{T}$

where

$\mathbf{R}_{d}=\left[\begin{array}{ll}g\left(k_{3}, k_{2}, k_{1}\right) & g\left(k_{4}, k_{2}, k_{1}\right) \\ g\left(k_{3}, k_{1}, k_{2}\right) & g\left(k_{4}, k_{1}, k_{2}\right)\end{array}\right]$,

where

$g(a, b, c)=-\frac{T\left(i a b^{2}-i a^{3}\right)+D_{1}(a / b-1)}{T\left(i c b^{2}-i c^{3}\right)+D_{1}(c / b-1)}$

and $D_{1} \equiv-i L_{2} \omega_{f}\left(12 / R-i \omega_{f}\right)$.

We define

$\left[A_{1}^{\prime \prime}, A_{2}^{\prime \prime}\right]^{T}=\mathbf{G}\left[A_{1}, A_{2}\right]^{T}$,

where $\mathbf{G}=\mathbf{R}_{d} \mathbf{P}_{d} \mathbf{R}_{u} \mathbf{P}_{u}$ denotes the $2 \times 2$ gain matrix describing the total change in amplitude of the upstream waves due to propagation along the channel and reflection at the boundaries.

On the mode- 1 stability boundary, $\mathbf{G}$ will have a unit eigenvalue (as there should be no net gain in amplitude); this can be interpreted as a resonance condition. Using the corresponding eigenvector as an initial amplitude for the two upstream waves, the two upstream waves observed after propagation along the channel and reflection at both boundaries are identical to the initial waves. This is illustrated in Fig. 8 for our chosen parameter combination on the mode-1 stability boundary (Fig. 7), where (a) shows the profile of the upstream waves, (b) shows the profile of the reflected downstream waves and (c) shows their superposition forming a mode-1 oscillation.

Using the properties of matrix norms $[45,49]$ it follows that

$\|\mathbf{G}\|_{2} \leqslant\left\|\mathbf{R}_{d}\right\|_{2}\left\|\mathbf{P}_{d}\right\|_{2}\left\|\mathbf{R}_{u}\right\|_{2}\left\|\mathbf{P}_{u}\right\|_{2}$,

where $\|.\|_{2}$ represents the two-norm of the matrix. Also, the eigenvalues $\lambda$ of $\mathbf{G}$ satisfy $\|\lambda\|_{2}<\|\mathbf{G}\|_{2}$ [50]. We define

$\mathcal{R} \equiv\left\|\mathbf{R}_{d}\right\|_{2}\left\|\mathbf{R}_{u}\right\|_{2}, \quad \mathcal{S} \equiv\left\|\mathbf{P}_{d}\right\|_{2}\left\|\mathbf{P}_{u}\right\|_{2}$,

so that $\|\lambda\|_{2} \leqslant \mathcal{R} \mathcal{S}$, where $\mathcal{R}$ denotes the contribution of wave reflections and $\mathcal{S}$ denotes the contribution of wave propagations. On the mode- 1 stability boundary, $\mathcal{R S} \geqslant 1$, as $\mathbf{G}$ has a unit eigenvalue. For our chosen parameter combination on this stability boundary (Fig. 7, Fig. 8) we find both $\left\|\mathbf{P}_{u}\right\|_{2}<1$ and $\left\|\mathbf{P}_{d}\right\|_{2}<1$ so $\mathcal{S}<1$ and wave propagations damp the amplitude of the wave profiles. Correspondingly $\mathcal{R}>1$, implying that amplification of the wave profiles at the boundaries is necessary to generate a neutrally stable oscillation.

The local analysis of Section 4.1 predicts potential growth of downstream-propagating wavepackets and therefore we might expect that downstream propagation would spatially amplify the wave. In Appendix B we consider forcing the system with fixed frequency. Choosing this forcing frequency to correspond to that of the neutrally stable mode- 1 oscillations, the contribution to the wavepacket in the laboratory frame due to the forcing (ignoring the contribution due to the local instability, as this is convected away) is attenuated in both directions (Fig. 9). Thus, for the parameters investigated, the component waves that constitute the mode1 oscillations are locally stable and the global instability arises due to amplification at the boundaries. 

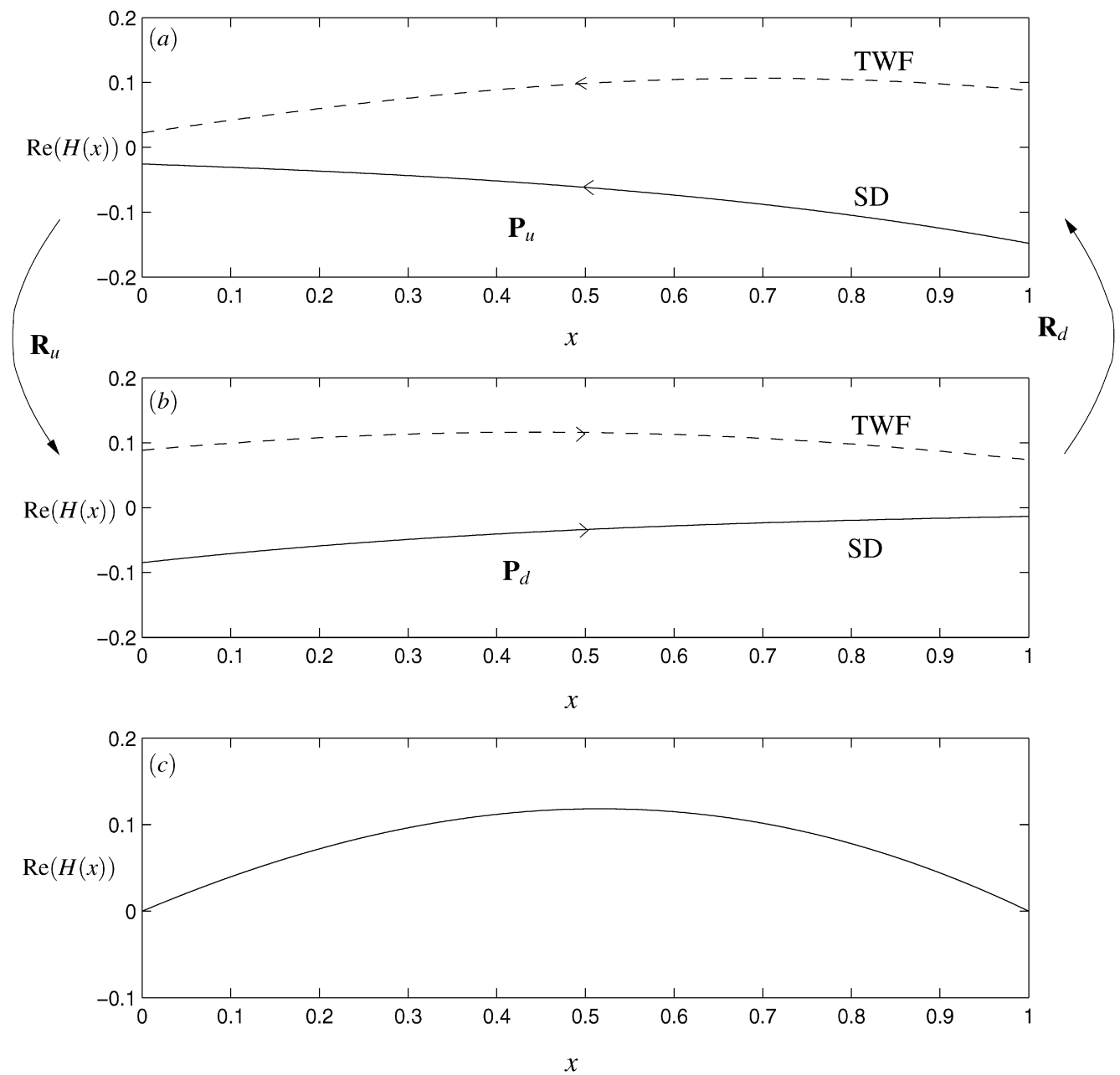

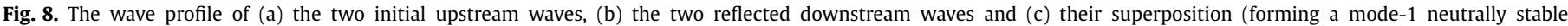
oscillation), illustrated at $t=0$. Here $T=10, R=15.9055, d=0, L_{1}=1, L_{2}=10$ and $\omega_{f}=-9.956$.

\section{Finite-amplitude oscillations}

We now consider finite-amplitude oscillations for zero wall damping $(d=0)$. We validate our weakly non-linear analysis for large $T$ describing the onset of mode- 1 oscillations (Appendix A), present a numerical sweep across parameter space along the two arrows shown in Fig. 3(b) and then compare energy budgets of mode- 1 and mode- 2 oscillations. In the following we define the time average of a quantity $g$ over a period of oscillation $\mathcal{T}_{1}$ as

$\bar{g}=\frac{1}{\mathcal{T}_{1}} \int_{t}^{t+\mathcal{T}_{1}} g \mathrm{~d} t^{\prime}$.

\subsection{Fully non-linear instabilities}

We illustrate the various oscillatory and static instabilities for $T=10$ (Fig. 10) and $T=0.3$ (Fig. 11). Given the deficiencies of the integral approximation, we choose not to dwell on details but instead seek to elucidate the overall characteristics of the system that may be shared by more sophisticated models.

Periodic solutions for $T=10$ are illustrated in Fig. 10, with $R$ increasing as indicated in the right-hand arrow in Fig. 3(b). The uniform state $h=1, q=1$ is stable until $R$ increases through 15.92 (linear stability predicts $R_{1}=15.91$ ). Here an oscillatory mode- 1 solution bifurcates subcritically from the uniform state, as predicted by the weakly non-linear analysis (Appendix A). This branch

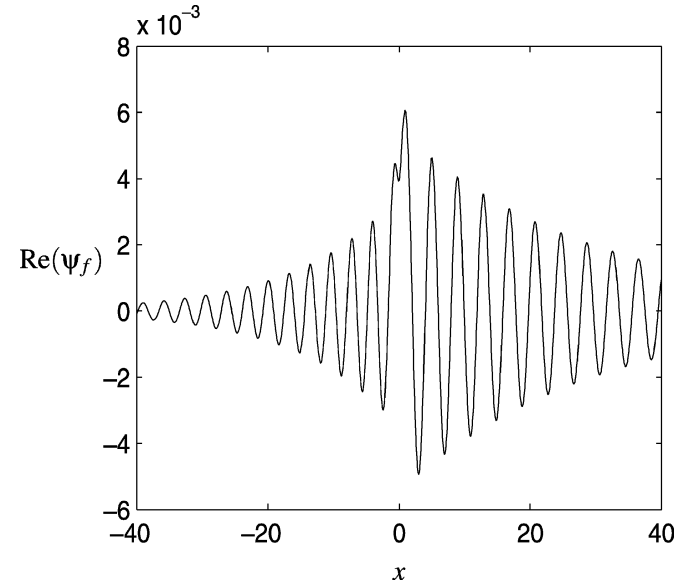

Fig. 9. The response to forcing at constant frequency, denoted $\psi_{f}$ and given by (B.5) Here $T=10, R=15.9055, d=0$ and $\omega_{f}=-9.956$.

is unstable, and thus difficult to track numerically, but some points are illustrated in Fig. 10(a), with an illustrative mode-1 oscillation for $R=15.0$ shown in Fig. 10(b-e). Increasing $R$ beyond 15.92, the steady solution jumps to a highly non-linear branch of stable periodic oscillations. This can be tracked back to $R \approx 13.2$, where it connects to the subcritical mode- 1 oscillatory branch. These oscillations exhibit a vigorous sloshing motion superimposed on the 

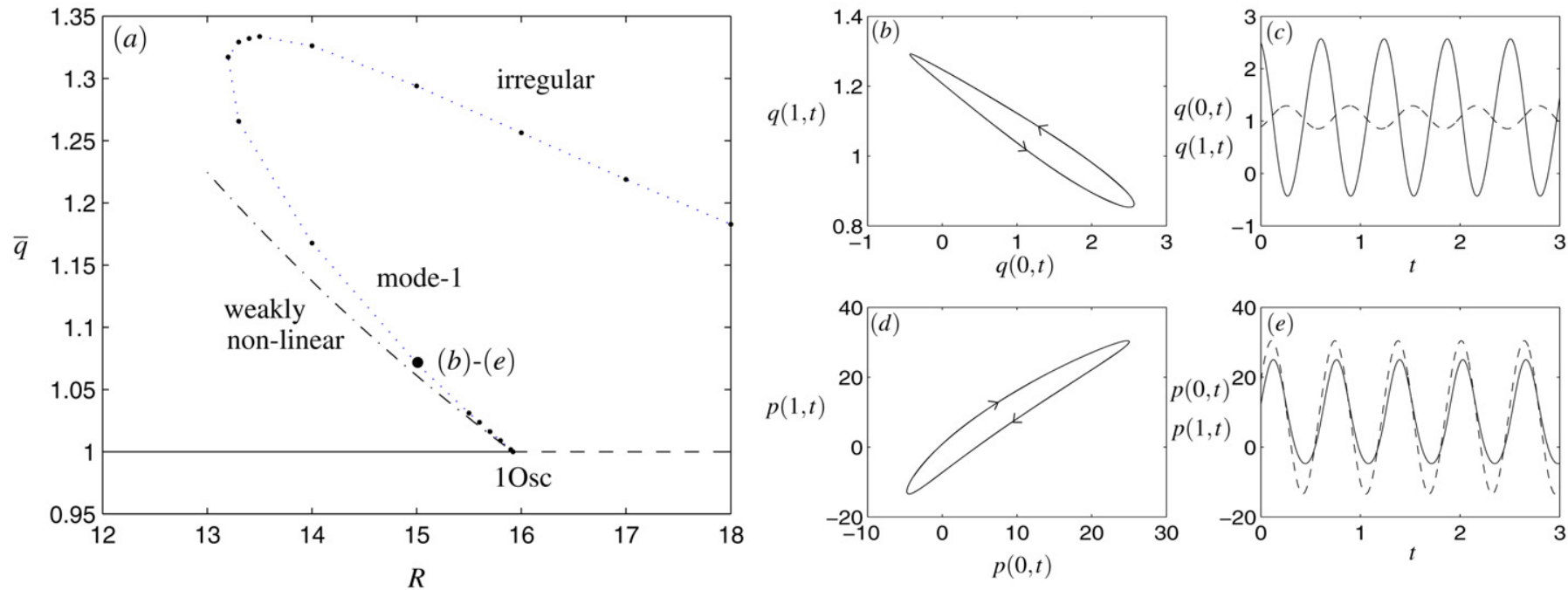

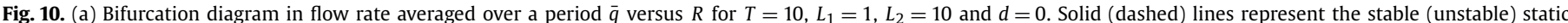

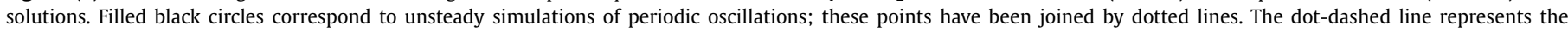

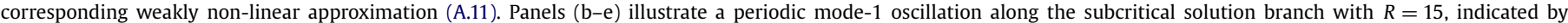

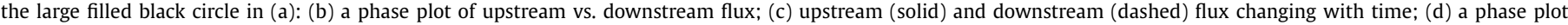
of upstream vs. downstream pressure; (e) upstream (solid) and downstream (dashed) pressure changing with time.
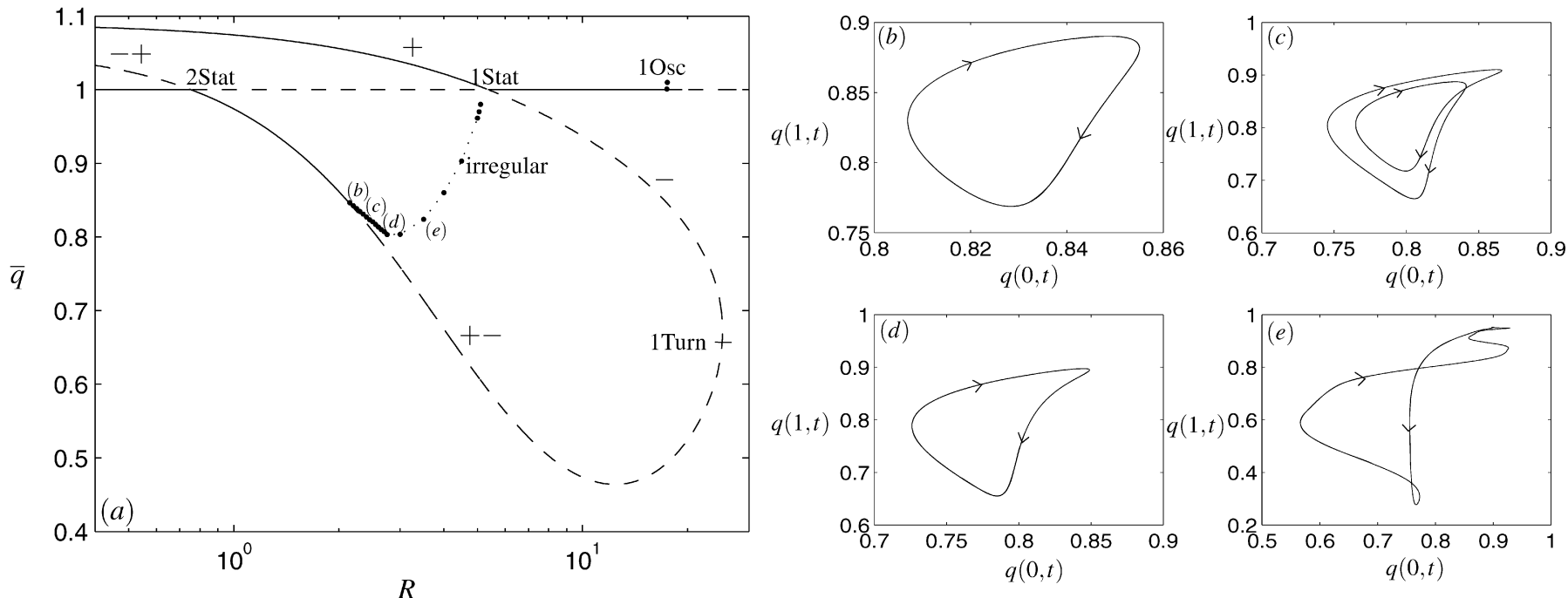

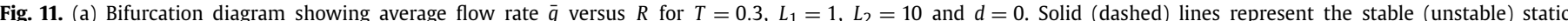

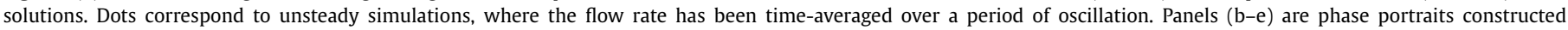
using up- and downstream flow rates, for (b) $R=2.3$, (c) $R=2.6$, (d) $R=2.8$ and (e) $R=3.6$.

mean flow. For the parameters chosen in Fig. 10(b-e), this sloshing is sufficient to cause transient flow reversal $(q<0)$ at the upstream end of the channel for a small portion of the oscillation.

The numerically determined eigenfunction for $T=10, R=$ 15.9 (appropriately normalised) is compared to the large-tension asymptotic approximation in Fig. 4, showing excellent agreement.

We now set $T=0.3$ and increase $R$ along the left-hand arrow in Fig. 3(b). The uniform state is stable for very low $R$ (Fig. 11(a)). As $R$ increases through $R \approx 0.75$ a stable mode- 2 static solution bifurcates from the uniform state, where the channel is inflated upstream and collapsed downstream, denoted ' +- '. This solution is stable until $R \approx 2.1$, when a mode- 2 oscillation develops through a supercritical Hopf bifurcation. A phase-portrait of the oscillation is shown in Fig. 11(b). This grows in amplitude until a period-doubling bifurcation occurs beyond $R \approx 2.4$ (Fig. 11(c)). This oscillation returns to a single period mode- 2 oscillation beyond $R \approx 2.8$ (Fig. 11(d)), becoming highly irregular as $R$ increases (Fig. 11(e)). The period of these oscillations increases dramatically as $\bar{q} \rightarrow 1$, an indication of a possible homoclinic bifurcation (reported in this context in [9]). In addition, we have observed other branches of irregular oscillations at higher Reynolds numbers, but we do not report these here. A mode- 1 static solution also bifurcates transcritically from the uniform state at $R \approx 5.36$. An unstable collapsed mode-1 static solution (denoted '-' in Fig. 11(a)) connects to the unstable static mode-2 branch at the point '1Turn'. In addition, a locally stable inflated mode- 1 static solution (denoted ' + ' in Fig. 11(a)) exists for $R \leqslant 5.36$.

As $R$ increases through 17.5 , an oscillatory mode- 1 solution bifurcates supercritically from the uniform state; a detailed bifurcation diagram was not constructed in this case. It is only at higher values of $T$ that this transition becomes subcritical (Fig. 10).

\subsection{Energy budget}

The finite-amplitude oscillations can be understood more clearly by examining the energy budget. 
When $d=0$, the energy budget (2.15) averaged over one period of oscillation takes the form

$\overline{\mathcal{F}}+\overline{\mathcal{P}}=\overline{\mathcal{D}}$,

since $\mathcal{K}$, the rate of change of kinetic energy, will average to zero over a period, as will $\mathcal{E}$, the rate of working of pressure forces across the membrane, because (in the absence of wall damping) it can be expressed as an exact derivative as

$\mathcal{E}=\frac{6}{10}\left(\int_{0}^{1} T h_{x}^{2} \mathrm{~d} x\right)_{t}+\frac{6}{5}\left(\int_{0}^{1}\left(p_{u}-\frac{12}{R}\left(x+L_{1}\right)\right) h \mathrm{~d} x\right)_{t}$.

Also, the time-averaged rate of working of pressure forces upstream takes the simple form

$\overline{\mathcal{P}}=\frac{6}{5} p_{u} \bar{q}$,

where $p_{u}=12\left(1+L_{1}+L_{2}\right) / R$.

Considering the mode- 1 oscillation at $T=10$ and $R=15$ (Fig. 10(b-e)), numerical values for the time-averaged quantities in the energy equation at different points along the bifurcation diagram are given in Table 1 . We also present the equivalent results

Table 1

Numerical values of time-averaged quantities in the energy equation.

\begin{tabular}{lllllll}
\hline$T$ & $R$ & Behaviour & $\bar{q}$ & $\overline{\mathcal{F}}$ & $\overline{\mathcal{P}}$ & $\overline{\mathcal{D}}$ \\
\hline 0.3 & 0.7 & uniform & 1.0 & 0.0 & 246.8571 & 246.8571 \\
0.3 & 1.5 & mode-2 stat & 0.9181 & 0.0 & 105.7688 & 105.7639 \\
0.3 & 2.3 & mode-2 osc (period 1) & 0.8347 & -0.0027786 & 62.7147 & 62.7118 \\
0.3 & 2.6 & mode-2 osc (period 2) & 0.8135 & -0.0073502 & 54.1063 & 54.0986 \\
0.3 & 2.8 & mode-2 osc (period 1) & 0.7993 & -0.0090417 & 49.328 & 49.3188 \\
10.0 & 15.0 & uniform & 1.0 & 0.0 & 11.52 & 11.52 \\
10.0 & 15.0 & mode-1 osc & 1.0712 & 2.5324 & 12.3387 & 14.8676 \\
10.0 & 15.0 & irregular & 1.2941 & 27.6082 & 14.8534 & 42.4688 \\
\hline
\end{tabular}
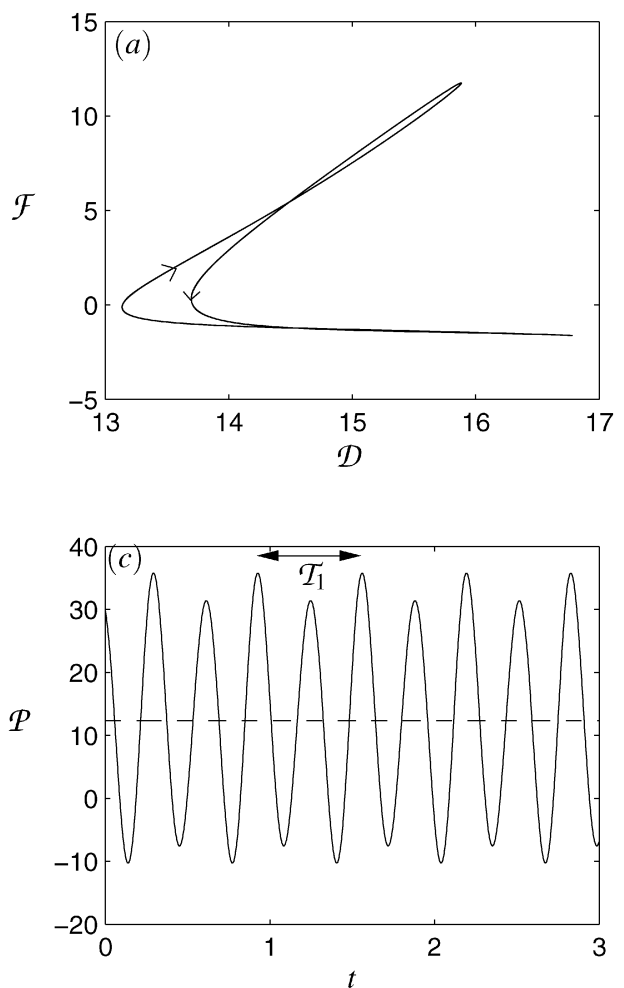

for the uniform base state, where time averaging is of course unnecessary. We estimate the numerical error by calculating $\overline{\mathcal{K}}$ and $\overline{\mathcal{E}}$, which should be zero over a period of oscillation. The absolute value of both these quantities is $O\left(10^{-3}\right)$, significantly smaller than each of the quantities under consideration. For mode- 1 oscillations about the uniform base state, the time-averaged net kinetic energy flux into the system, $\overline{\mathcal{F}}$, is positive, showing that kinetic energy is extracted from the mean flow and is dissipated by the oscillations (as found by Jensen and Heil [19] in the full twodimensional system).

We illustrate the phase portrait of net kinetic energy flux $(\mathcal{F})$ against the rate of energy loss due to viscous dissipation $(\mathcal{D})$ in Fig. 12(a), along with the temporal evolution of each of the quantities in the energy equation (5.1) in Fig. 12(b-d), for the small amplitude mode-1 oscillation shown in Fig. 10(b-e). In regions where $\mathcal{F}>0(\mathcal{F}<0)$, upstream sloshing motion reinforces (opposes) the mean flow. The rate of viscous dissipation is minimised at times when the kinetic energy flux is close to zero, so $\mathcal{D}$ and $\mathcal{P}$ have two local minima over one period of oscillation. Kinetic energy is extracted from the mean flow for approximately half the period of oscillation (yielding $\overline{\mathcal{F}}>0$ ); there is also a net increase in the flow rate along the channel $(\bar{q}>1)$, which (5.3) shows is destabilising by increasing $\overline{\mathcal{P}}$.

For the oscillation bifurcating from the mode-2 static solution at low tension, $T=0.3$, numerical values for the time-averaged quantities in the energy equation at different points along the bifurcation diagram in Fig. 11 are also given in Table 1. The absolute values of both $\overline{\mathcal{K}}$ and $\overline{\mathcal{E}}$ are $O\left(10^{-4}\right)$, at least an order of magnitude smaller than each of the quantities under consideration. In this case the net kinetic energy flux into the system over one oscillation is slightly negative, so a small amount of kinetic energy is extracted from the oscillation by the mean flow. Oscillations are instead driven entirely by the rate of working of pressure forces upstream (5.3). We illustrate the phase portrait of net kinetic energy flux $(\mathcal{F})$ against the rate of energy loss due to viscous dissi-
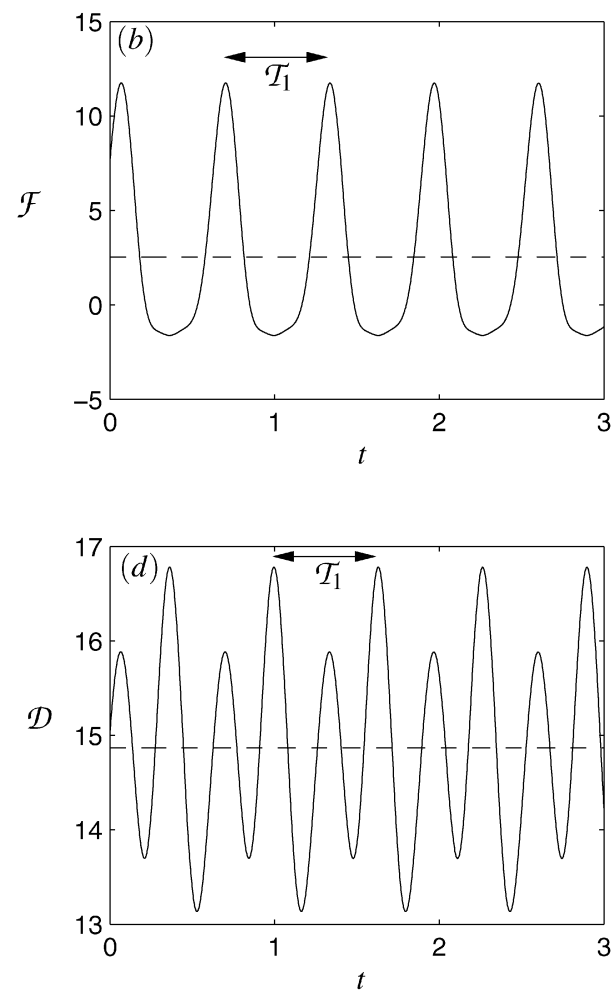

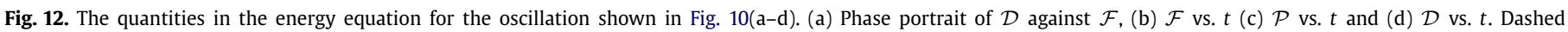
lines denote the time-averaged values of the quantities over a period of oscillation $\mathcal{T}_{1}$. Here $T=10, R=15, d=0, L_{1}=1, L_{2}=10$. 

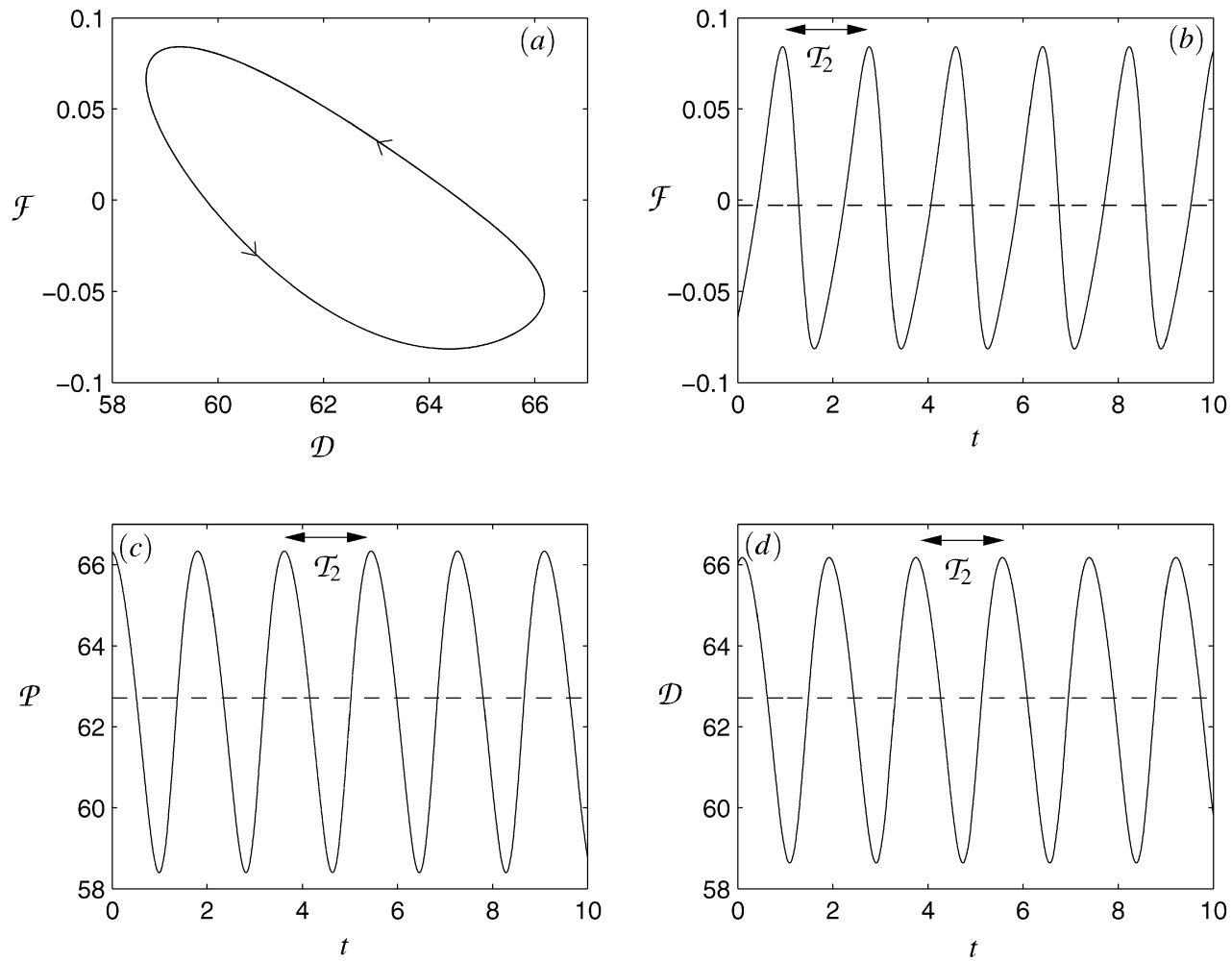

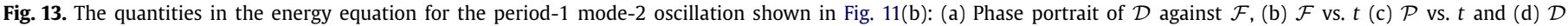
vs. $t$. Dashed lines denote the time-averaged values of the quantities over a period of oscillation $\mathcal{T}_{2}$. Here $T=0.3, R=2.3, d=0, L_{1}=1, L_{2}=10$.

pation $(\mathcal{D})$ (Fig. 13(a)), along with the temporal evolution of each of the quantities in the energy equation (Fig. 13(b-d)), for the oscillation shown in Fig. 11(a). The phase portrait differs significantly from that shown in Fig. 12(b), where now the rate of energy loss due to viscous dissipation and the net kinetic energy flux have the same period as the oscillation, but are out of phase.

Thus differences in the energy budget between these two small-amplitude oscillations, mode- 1 (about the uniform base state) and mode-2 (about a non-uniform base state) are notable, both in the signature of energy transport (Fig. 12(a), Fig. 13) and in the sign of kinetic energy flux $\overline{\mathcal{F}}$ (Table 1 ), which does not appear to change at larger amplitudes. We conjecture that these differences reflect fundamentally different mechanisms of oscillation.

\section{Discussion}

In this paper we have developed a spatially one-dimensional model of flow in a long flexible-walled channel, using an integral approximation of the boundary-layer equations that takes full account of experimentally realistic boundary conditions. The model has a number of obvious limitations, for example in not describing accurately high-frequency, high-Reynolds-number flows, and in failing to capture short-wavelength hydrodynamic instabilities such as Tollmien-Schlichting waves. Nevertheless, the simplicity of the model yields valuable insights that can be used in more sophisticated approaches. In particular, the model's evolution equations (2.11) are numerically relatively straightforward to handle, providing us with a useful overview of parameter space, although quantitative details (for example of highly non-linear oscillations) should be interpreted with caution. We have chosen here to use a very simple wall model (2.6), ignoring wall mass (and therefore some flutter modes), wall bending stresses and wall stretching. In addition, we chose an external pressure distribution that yields a uniform base state. This is useful in allowing a straightforward stability analysis but it introduces a symmetry to the base state that is not generic. In particular, the transcritical bifurcations of the uniform state to static deformed states (e.g. Fig. 11) are not structurally stable and disconnected solution branches can be anticipated, as seen in [42].

Linear stability analysis of the uniform state reveals families of static and oscillatory instabilities (Fig. 3). The static instabilities are analogous to those identified previously in both one and two-dimensional models [11,42]. In particular, we note that the channel readily adopts the mode-2 static configuration (Fig. 11(a)) commonly seen when the external pressure is uniform, with the channel inflated at its upstream end and collapsed further downstream. Multiple oscillatory modes have also been identified previously in one- and two-dimensional models of collapsible tube systems that impose uniform external pressure $[12,18]$. We examined in particular two classes of oscillatory instability: the mode-1 instability of the uniform base state that arises at high wall tension (Fig. 10); and the instability of the mode- 2 steady state arising at low tension (Fig. 11).

The mode- 1 instability was described in detail previously using both asymptotics and Navier-Stokes simulations in a twodimensional model by Jensen and Heil [19]; their work provides a useful test of the validity of the present one-dimensional model. They showed that a spatially asymmetric normal mode can extract energy from the mean flow by virtue of the kinetic energy flux at the upstream end of the collapsible segment exceeding the outward flux at the downstream end. Here we have developed the analogous asymptotic analysis in the large-tension limit of the onedimensional model. Because the description of viscous dissipation in (2.11) is inaccurate at high frequencies, failing to capture thin viscous boundary layers, the present one-dimensional model fails to capture the correct $T_{0}^{1 / 4}$ scaling of the critical Reynolds number with tension [19]. Nevertheless many other features of the instability are recovered; the leading-order inviscid normal mode (Fig. 4) is identical, for example. We have shown how, at finite amplitudes, 

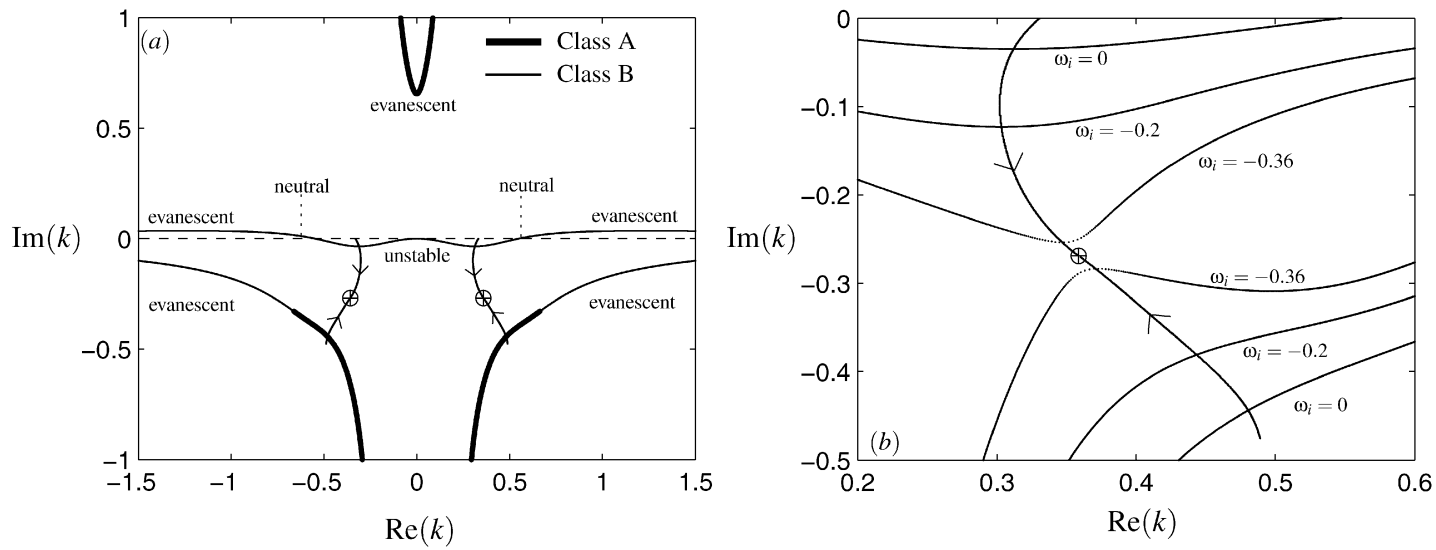

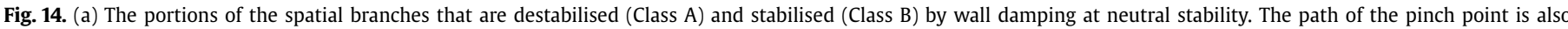

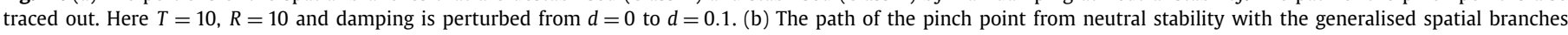
for $\omega_{i}=0,-0.2,-0.36$ for $T=10, R=10, d=0$.

Reynolds stresses increase the mean flux driven by the fixed imposed pressure drop (Appendix A), thereby extracting additional energy from the mean flow by increasing the rate at which the imposed pressure drop does work on the channel (see Section 5.2). This causes the oscillation to arise subcritically (Fig. 10(a)). The time-averaged energy budget (5.1) for a weakly non-linear mode-1 oscillation can be written in the form

$\overline{\mathcal{D}}_{S}+\overline{\mathcal{D}}_{P}+\overline{\mathcal{D}}_{N}=\overline{\mathcal{P}}+\overline{\mathcal{F}}$

where $\overline{\mathcal{D}}_{P}$ and $\overline{\mathcal{D}}_{S}$ represent the energy lost due to viscous dissipation in the mean flow and the oscillation respectively and $\overline{\mathcal{D}}_{N}$ represents the energy loss due to the prescribed flow profile being non-conservative. For an oscillation of small amplitude $A$ (see (A.5), (A.6)), the leading order energies take the form

$\overline{\mathcal{F}}=\frac{27}{25} A^{2} q_{00}\left(Q_{0}^{2}(0)-Q_{0}^{2}(1)\right)$,

$\overline{\mathcal{P}}=\frac{72}{5 R} q_{00}\left(1+L_{1}+L_{2}\right)$,

$\overline{\mathcal{D}}_{P}=\frac{72}{5 R} q_{00}^{2}\left(1+L_{1}+L_{2}\right)$

$\overline{\mathcal{D}}_{S}=\frac{6}{10} A^{2}\left[\left\langle Q_{0}, Q_{0}\right\rangle+L_{1} Q_{0}^{2}(0)+L_{2} Q_{0}^{2}(1)\right]$,

$\overline{\mathcal{D}}_{N}=-\frac{3}{50} A^{2} q_{00}\left(Q_{0}^{2}(0)-Q_{0}^{2}(1)\right)$

Using the critical Reynolds number $R_{1}$ (3.16), at criticality

$\frac{\overline{\mathcal{D}}_{S}}{\overline{\mathcal{F}}}=\frac{2}{3}$.

Thus, $2 / 3$ of the energy extracted from the mean flow is lost to viscous dissipation in the oscillation with the remainder balanced by increased dissipation in the mean flow. This result was first shown by Jensen and Heil [19] for the two-dimensional system. We note that

$\frac{\overline{\mathcal{D}}_{N}}{\overline{\mathcal{F}}}=-\frac{1}{18}$,

and so the energy gained due to our flow profile assumption (Section 2.2) is only a small fraction of the net kinetic energy extracted from the mean flow, and makes no qualitative difference to the results.

The mode-2 instability bifurcating from a non-uniform base state (Fig. 11) resembles qualitatively that described in other onedimensional models $[13,15]$, two-dimensional simulations $[17,18]$ and, to some extent, experiments [3,4]. Significantly, we have shown that mode-2 oscillations have a qualitatively different energy balance from mode-1: in particular, mode- 2 oscillations do not extract energy from the mean flow via kinetic energy fluxes (Table 1). There is therefore not the clear link identified in [19] for mode- 1 between instability and the asymmetric boundary conditions. Instead, the primary source of instability for mode- 2 appears to be the enhanced rate of working of pressure forces by adjustments to the mean flux, presumably via the action of Reynolds stresses.

We have also used the simple structure of the uniform base state to explore the connections between local and global instabilities. In the absence of wall damping, we found that the uniform base state can be convectively, but not absolutely, unstable (for the parameter values that we investigated). The convective instability arises through the merger of unstable downstream-propagating travelling-wave flutter and upstream-propagating static-divergence (Fig. 14). Absolute instability can be expected in the presence of wall damping, as noted for example by Wiplier and Ehrenstein [51], who found absolute instability in flow over a compliant damped membrane formed by the merging of an unstable Tollmien-Schlichting wave and an evanescent wave (see also [6]); preliminary calculations indicate that absolute instability arises in the present model in the presence of sufficient wall damping. Further, we showed how the mode- 1 instability can arise in a region of parameter space that is convectively stable, demonstrating that convective instability is not a necessary condition for oscillations. Temporal instability in a finite-length system, for which the infinite-length system is convectively stable was also identified by Doaré and de Langre [52] in the case of a cantilevered flexible pipe on an elastic foundation, and by Peake [46], who showed how resonance conditions can be defined for flow over a finitelength flexible plate by examining the propagation and reflection of waves having wavelengths shorter than the plate. In the present case, fragments of long individual waves (travelling-wave flutter and static-divergence) combine in a non-trivial way to produce the resonant mode-1 oscillatory instability (Fig. 8), amplifying via wave reflections at the boundaries with the rigid parts of the system. Decomposing the oscillatory instability of the non-uniform mode2 steady state (e.g. that shown in Fig. 11(b)) in a similar manner remains an open problem.

In summary, we have demonstrated that local analysis gives only limited insight into the properties of the global system, because wave reflections at boundaries play a vital role in the growth of mode- 1 oscillations. Further, we have found that these mode- 1 disturbances can extract energy from the mean flow in a notably different manner to the mode- 2 disturbances that emerge from a non-uniform base state, exhibiting strikingly different energy sig- 
natures (Figs. 12(a), 13(a)). It remains now to test these results against more accurate two- and three-dimensional models and to consider the role of the additional hydrodynamic disturbances present in these systems.

\section{Acknowledgements}

PSS acknowledges support from BBSRC. SLW acknowledges support from the EPSRC in the form of an Advanced Research Fellowship. The authors are grateful to J. Billingham, R.J. Whittaker, M. Heil and J.W. Boyle for useful comments and discussions.

\section{Appendix A. Weakly non-linear stability with $T \gg 1$}

The large-tension limit described in Section 3.4, for which we used an expansion in $T^{-1 / 2}$ to understand the linear stability problem, can be extended further to capture leading-order finiteamplitude effects.

We return to the full model (2.11), and rescale using

$t=\delta_{1} \tilde{t}, \quad h=1+\delta_{1} \tilde{h}$,

where $\delta_{1}=T^{-1 / 2}$. Dropping tildes, we assume a regular perturbation expansion for each of the variables in the form

$q=q_{0}+\delta_{1} q_{1}+\cdots, \quad h=h_{0}+\delta_{1} h_{1}+\cdots$,

and introduce slow time variables $t_{j}=t \delta_{1}^{j}, j=1,2, \ldots$. At leading order

$h_{0 t}+q_{0 x}=0, \quad q_{0 t}-h_{0 x x x}=0 \quad(0 \leqslant x \leqslant 1)$,

$h_{0}=0, \quad h_{0 x x}-L_{1} q_{0 t}=0 \quad(x=0)$

$h_{0}=0, \quad h_{0 x x}+L_{2} q_{0 t}=0 \quad(x=1)$,

and at the following order

$h_{0 t_{1}}+h_{1 t}+q_{1 x}=0$,

$q_{0 t_{1}}+q_{1 t}-h_{1 x x x}=-\frac{12}{5} q_{0} q_{0 x}+h_{0} h_{0 x x x}+\frac{12}{R}\left(1-q_{0}\right)$

$(0 \leqslant x \leqslant 1)$

$h_{1}=0, \quad h_{1 x x}-L_{1} q_{1 t}-L_{1} q_{0 t_{1}}=\frac{12 L_{1}}{R}\left(q_{0}-1\right) \quad(x=0)$,

$h_{1}=0, \quad h_{1 x x}+L_{2} q_{1 t}+L_{2} q_{0 t_{1}}=-\frac{12 L_{2}}{R}\left(q_{0}-1\right)$

$(x=1)$.

The leading-order problem has the solution

$q_{0}=q_{00}+A\left(t_{1}, t_{2}, \ldots\right) q_{01} \cos \beta_{1} t$

$h_{0}=A\left(t_{1}, t_{2}, \ldots\right) h_{01} \sin \beta_{1} t$,

where $q_{00}$ is some constant uniform mean flow that is not determined at leading order. $A\left(t_{1}, t_{2}, \ldots\right)$ is a slowly varying oscillatory amplitude and

$q_{01}=Q_{0}, \quad h_{01}=-q_{01 x} / \beta_{1}$,

where $Q_{0}$ is the real eigenfunction determined in Section 3.4.

The forcing terms at second order suggest that

$q_{1}=q_{10}+A q_{11} \sin \beta_{1} t+A^{2} q_{12} \sin 2 \beta_{1} t$,

$h_{1}=h_{10}+A h_{11} \cos \beta_{1} t+A^{2} h_{12} \cos 2 \beta_{1} t$.

Taking the steady component of (A.4a), we have $q_{10 x}=0$ and then (A.4b)-(A.4d) give

$$
\begin{aligned}
& -h_{10 x x x}=-\frac{6}{5} A^{2} q_{01} q_{01 x}+\frac{1}{2} A^{2} h_{01} h_{01 x x x}+\frac{12}{R}\left(1-q_{00}\right) \\
& \quad(0 \leqslant x \leqslant 1), \\
& h_{10}=0, \quad h_{10 x x}=\frac{12 L_{1}}{R}\left(q_{00}-1\right) \quad(x=0), \\
& h_{10}=0, \quad h_{10 x x}=-\frac{12 L_{2}}{R}\left(q_{00}-1\right) \quad(x=1) .
\end{aligned}
$$

Thus

$h_{10 x x x}=\frac{7 A^{2}}{20}\left(Q_{0}^{2}\right)_{x}+\frac{12}{R}\left(q_{00}-1\right)$.

Integrating once, applying the two boundary conditions on $h_{10 x x}$, we obtain the constraint

$q_{00}=1+\frac{7 R A^{2}}{240\left(1+L_{1}+L_{2}\right)}\left[Q_{0}^{2}(0)-Q_{0}^{2}(1)\right]$

Non-linear Reynolds stresses generate a pressure gradient and since both the upstream and downstream pressures are prescribed, the mean flow in the channel must adjust. Since $Q_{0}^{2}(0)-Q_{0}^{2}(1)>$ 0 , the mean flow rate along the channel $\left(q_{00}\right)$ increases and so the oscillation acts as a pump.

The component of (A.4) with frequency $\beta_{1}$ gives

$$
\begin{aligned}
& -A \beta_{1} h_{11}+A q_{11 x}=\frac{q_{01 x}}{\beta_{1}} \frac{\partial A}{\partial t_{1}}, \\
& \beta_{1} A q_{11}-A h_{1 x x x}=-q_{01} \frac{\partial A}{\partial t_{1}}-\frac{12}{5} A q_{00} q_{01 x}-\frac{12}{R} A q_{01}, \\
& h_{11}=0, \quad A h_{11 x x}-A L_{1} \beta_{1} q_{11}=L_{1} q_{01}\left[\frac{12}{R} A+\frac{\partial A}{\partial t_{1}}\right] \\
& \quad(x=0), \\
& h_{11}=0, \quad A h_{11 x x}+A L_{2} \beta_{1} q_{11}=-L_{2} q_{01}\left[\frac{12}{R} A+\frac{\partial A}{\partial t_{1}}\right] \\
& \quad(x=1) .
\end{aligned}
$$

Applying the solvability condition (3.15), we obtain

$$
\begin{aligned}
& \left(\frac{12 A}{R}+\frac{\partial A}{\partial t_{1}}\right)\left[L_{1} Q_{0}^{2}(0)+L_{2} Q_{0}^{2}(1)+\left\langle Q_{0}, Q_{0}\right\rangle\right] \\
& \quad=\frac{12 q_{00} A}{10}\left[Q_{0}^{2}(0)-Q_{0}^{2}(1)\right] .
\end{aligned}
$$

For $A \rightarrow 0, q_{00} \rightarrow 1$ and we recover the critical Reynolds number $R_{1}$ given in (3.16). Substituting (A.11) into (A.13) gives

$\frac{\partial A}{\partial t_{1}}=12 A\left(\frac{R}{R_{1}}\right)\left(\frac{7 A^{2}\left[Q_{0}^{2}(0)-Q_{0}^{2}(1)\right]}{240\left(1+L_{1}+L_{2}\right)}-\frac{R_{1}-R}{R^{2}}\right)$.

Thus, for a constant amplitude oscillation, either $A=0$ or

$A^{2}=\frac{240\left(1+L_{1}+L_{2}\right)}{7\left[Q_{0}^{2}(0)-Q_{0}^{2}(1)\right]}\left(\frac{R_{1}-R}{R^{2}}\right)$,

indicating that periodic solutions bifurcate subcritically from the uniform state as $R$ increases through $R_{1}\left(A^{2}>0\right.$ for $R<R_{1}$, under the necessary condition $Q_{0}^{2}(0)>Q_{0}^{2}(1)$ ).

\section{Appendix B. Signalling and mode classification}

We now consider the signalling problem (forcing the system with fixed frequency $\left.\omega_{f}\right)[30,31]$ in order to classify the waves contributing to the local convective instability and to the global mode-1 oscillatory instability. We set

$\mathcal{D}_{1}\left[\begin{array}{l}\hat{h} \\ \hat{q}\end{array}\right]=\left[\begin{array}{c}0 \\ \delta(x) H(t) \mathrm{e}^{-i \omega_{f} t}\end{array}\right]$, 
where $H(t)$ is a Heaviside unit-step function. Taking a FourierLaplace transform according to

$\tilde{\tilde{f}}(k, \omega)=\int_{0}^{\infty} \int_{-\infty}^{\infty} \mathrm{e}^{-i(k x-\omega t)} f(x, t) \mathrm{d} x \mathrm{~d} t$,

the forced governing equations become

$D(k, \omega) \tilde{\tilde{\psi}}=\frac{i}{\left(\omega-\omega_{f}\right)}$,

where $\psi$ is the response to forcing with fixed frequency. This response has two components: the first equivalent to that for an impulse forcing, denoted $\psi_{s}$; and the second due to the signal, denoted $\psi_{f}$. In the laboratory frame $\psi_{s}$ will decay for large time (as the flow is convectively unstable) leaving only the component with fixed frequency $\omega_{f}$, given by

$\psi_{f}(x, t)=\frac{\mathrm{e}^{-i \omega_{f} t}}{2 \pi} \int_{F_{k}} \frac{\mathrm{e}^{i k x}}{D\left(k, \omega_{f}\right)} \mathrm{d} k$

Since the contour $L_{\omega}$ lies above all the poles in the $\omega$ plane, we choose the contour $F_{k}$ to lie along the real $k$ axis (with a suitable deformation around the branch cut, e.g. Fig. 6(a)), which will again split the generalised spatial branches into two disconnected sets. We denote the two spatial branches in the upper half plane as $k_{1+}\left(\omega_{f}\right)$ and $k_{2+}\left(\omega_{f}\right)$, while the two in the lower half plane are denoted $k_{1-}\left(\omega_{f}\right)$ and $k_{2-}\left(\omega_{f}\right)$.

We evaluate (B.4) using the residue theorem, and for $x>0(x<$ 0 ) we close the contour $F_{k}$ as a semi-circle from above (below) giving

$$
\begin{aligned}
\psi_{f}= & i H(x)\left(\frac{\mathrm{e}^{i\left(k_{1+} x-\omega_{f} t\right)}}{\frac{\partial D}{\partial k}\left[k_{1+}, \omega_{f}\right]}+\frac{\mathrm{e}^{i\left(k_{2+} x-\omega_{f} t\right)}}{\frac{\partial D}{\partial k}\left[k_{2+}, \omega_{f}\right]}\right) \\
& -i H(-x)\left(\frac{\mathrm{e}^{i\left(k_{1-}-\omega_{f} t\right)}}{\frac{\partial D}{\partial k}\left[k_{1-}, \omega_{f}\right]}+\frac{\mathrm{e}^{i\left(k_{2-}-\omega_{f} t\right)}}{\frac{\partial D}{\partial k}\left[k_{2-}, \omega_{f}\right]}\right),
\end{aligned}
$$

where $H(x)$ is again the Heaviside unit step function. $k_{1+}$ and $k_{2+}\left(k_{1-}\right.$ and $\left.k_{2-}\right)$ define waves that propagate downstream (upstream).

This classification holds when the contour $L_{\omega}$ is chosen above all the temporal singularities (e.g. Fig. 5). However, once the deformation of the contour $L_{\omega}$ requires a corresponding deformation in the contour $F_{k}$ (i.e. $\operatorname{Im}\left(\omega_{f}\right)<\max (\operatorname{Im}(\omega))$ for real $k$ ) we cannot simply look to $\operatorname{Im}(k)$ in general to decide if the wave is propagating upstream or downstream. Instead we follow the evolution of the spatial branches as $L_{\omega}$ is lowered towards the real axis. The direction of propagation of the wave is determined by the side of the $k$-plane from which the spatial branch originates $[44,45]$. Thus any portion of a spatial branch that has crossed the $\operatorname{Re}(k)$ axis from above defines a convectively unstable downstream wave, whilst portions that have not crossed the $\operatorname{Re}(k)$ axis are evanescent (Peake [46]). Fig. 14 illustrates how, for the example illustrated in Fig. 6(c), the disturbance is advected downstream of the point of excitation since only downstream propagating waves become unstable (in the laboratory frame).

These waves can be more broadly classified using a framework based on activation energy [32-34]. Upon adding a small amount of wall damping to the neutrally stable waves, those destabilised are Class A (or negative energy waves) whilst those stabilised are Class B (or positive energy waves) (Fig. 14). We find that the upstream spatial branches contain portions of both Class A and Class B waves, where the change in behaviour coincides with $\omega_{r}$ increasing through zero.

The unstable downstream waves are stabilised by wall damping and are thus Class B, classified as travelling-wave flutter (TWF)
$[5,47]$. To identify the class of the upstream evanescent wave at pinching we track the path of the wavenumbers that eventually pinch back to neutral stability [41], as follows. For $k$ real we identify the points in the $\omega$-plane where $\operatorname{Im}(\omega)$ is a maximum (Fig. 5). We then decrease the imaginary part of $k$ in small fixed steps and at each step identify the point in the $\omega$-plane where $\operatorname{Im}(\omega)$ is a maximum connected by continuity from the maximum observed at the previous step. Mapping from this $\omega$ we can track the four corresponding wavenumbers and plot their paths in the $k$-plane. The path of the two waves that eventually pinch are shown in Fig. 14(b). The upstream propagating waves at neutral stability in Fig. 14(a) are therefore Class A (static-divergence).

\section{References}

[1] M. Heil, O.E. Jensen, Flows in deformable tubes and channels, in: P.W. Carpenter, T.J. Pedley (Eds.), Flow Past Highly Compliant Boundaries and in Collapsible Tubes, Kluwer, 2003, pp. 15-49.

[2] J.B. Grotberg, O.E. Jensen, Biofluid mechanics in flexible tubes, Annu. Rev. Fluid Mech. 36 (2004) 121-147.

[3] C.D. Bertram, C.J. Raymond, T.J. Pedley, Mapping of instabilities for flow through collapsed tubes of different length, J. Fluids Struct. 4 (1990) 125-154.

[4] C.D. Bertram, C.J. Raymond, T.J. Pedley, Application of non-linear dynamics concepts to the analysis of self-excited oscillations of a collapsible tube conveying a fluid, J. Fluids Struct. 5 (1991) 391-426.

[5] C. Davies, P.W. Carpenter, Instabilities in a plane channel flow between compliant walls, J. Fluid Mech. 352 (1997) 205-243.

[6] C. Davies, Convective and absolute instabilities of flow over compliant walls, in: P.W. Carpenter, T.J. Pedley (Eds.), Flow Past Highly Compliant Boundaries and in Collapsible Tubes, Kluwer, 2003, pp. 69-93.

[7] T.J. Pedley, Longitudinal tension variation in a collapsible channel: a new mechanism for the breakdown of steady flow, Trans. ASME J. Biomech. Eng. 114 (1992) 60-67.

[8] C.D. Bertram, T.J. Pedley, A mathematical model of unsteady collapsible tube behaviour, J. Biomech. 19 (1982) 39-50.

[9] J.P. Armitstead, C.D. Bertram, O.E. Jensen, A study of the bifurcation behaviour of a model of flow through a collapsible tube, Bull. Math. Biol. 58 (1996) 611641.

[10] C. Cancelli, T.J. Pedley, A separated-flow model for collapsible-tube oscillations, J. Fluid Mech. 157 (1985) 375-404.

[11] O.E. Jensen, T.J. Pedley, The existence of steady flow in a collapsed tube, J. Fluid Mech. 206 (1989) 339-374.

[12] O.E. Jensen, Instabilities of flow in a collapsed tube, J. Fluid Mech. 220 (1990) 623-659.

[13] O.E. Jensen, Chaotic oscillations in a simple collapsible tube model, Trans. ASME J. Biomech. Eng. 114 (1992) 55-59.

[14] Y. Matsuzaki, T. Matsumoto, Flow in a two-dimensional channel with rigid inlet and outlet, Trans. ASME J. Biomech. Eng. 111 (1989) 180-184.

[15] S. Hayashi, T. Hayase, H. Kawamura, Numerical analysis for stability and selfexcited oscillation in collapsible tube flow, Trans. ASME J. Biomech. Eng. 120 (1998) 468-475

[16] S. Hayashi, T. Hayase, Y. Miura, I. Iimura, Dynamic characteristics of collapsible tube flow, JSME Int. J. Ser. C 42 (1999) 689-696.

[17] X.-Y. Luo, T.J. Pedley, A numerical simulation of unsteady flow in a twodimensional collapsible channel, J. Fluid Mech. 314 (1996) 191-225.

[18] X.-Y. Luo, Z.X. Cai, W.G. Li, T.J. Pedley, The cascade structure of linear instability in collapsible channel flows, J. Fluid Mech. 600 (2008) 45-76.

[19] O.E. Jensen, M. Heil, High-frequency self-excited oscillations in a collapsiblechannel flow, J. Fluid Mech. 481 (2003) 235-268.

[20] M. Heil, S.L. Waters, Transverse flows in rapidly oscillating elastic cylindrical shells, J. Fluid Mech. 547 (2006) 185-214.

[21] M. Heil, S.L. Waters, How rapidly oscillating collapsible tubes extract energy from a viscous mean flow, J. Fluid Mech. 601 (2008) 199-227.

[22] R.J. Whittaker, S.L. Waters, O.E. Jensen, J.W. Boyle, M. Heil, The energetics of flow through a rapidly oscillating flexible tube: Part I: General theory, 2008, submitted for publication.

[23] R.J. Whittaker, S.L. Waters, O.E. Jensen, J.W. Boyle, M. Heil, The energetics of flow through a rapidly oscillating flexible tube: Part II: Application to an elliptical tube, 2008, submitted for publication.

[24] A.L. Hazel, M. Heil, Steady finite-Reynolds-number flows in three-dimensional collapsible tubes, J. Fluid Mech. 486 (2003) 79-103.

[25] A. Marzo, X.Y. Luo, C.D. Bertram, Three-dimensional collapse and steady flow in thick-walled flexible tubes, J. Fluids Struct. 20 (2005) 817-835.

[26] M. Heil, A.L. Hazel, J. Boyle, Solvers for large-displacement fluid-structure interaction problems: segregated versus monolithic approaches, Comput. Mech. 43 (2008) 91-101. 
[27] X.-Y. Luo, T.J. Pedley, A numerical simulation of steady flow in a 2-D collapsible channel, J. Fluids Struct. 9 (1995) 149-197.

[28] X.-Y. Luo, T.J. Pedley, The effects of wall inertia on flow in a two-dimensional collapsible channel, J. Fluid Mech. 363 (1998) 253-280.

[29] T.R. Salamon, R.C. Armstrong, R.A. Brown, Traveling waves on vertical films: numerical analysis using the finite element method, Phys. Fluids 6 (1994) 2202 2220.

[30] P. Huerre, Spatio-temporal instabilities in open and closed flows, in: E. Tirapegui, D. Villaroel (Eds.), Instabilities and Nonequilibrium Structures, Reidel, Dordrecht, 1987, pp. 141-177.

[31] P. Huerre, M. Rossi, Hydrodynamic instabilities in open flows, in: C. Godrèche P. Manneville (Eds.), Hydrodynamics and Nonlinear Instabilities, Cambridge University Press, 1998, pp. 81-294.

[32] T.B. Benjamin, Effects of a flexible boundary on hydrodynamic stability, J. Fluid Mech. 9 (1960) 513-532.

[33] M.T. Landahl, On the stability of a laminar incompressible boundary layer over a flexible surface, J. Fluid Mech. 13 (1962) 609-632.

[34] T.B. Benjamin, The threefold classification of unstable disturbances in flexible surfaces bounding inviscid flows, J. Fluid Mech. 16 (1963) 436-450.

[35] V.Ya. Shkadov, Wave conditions in the flow of thin layer of a viscous liquid under the action of gravity, Izv. Akad. Nauk SSSR, Mekh. Zhidk. Gaza 1 (1967) 43-50.

[36] V.Ya. Shkadov, Theory of wave flows of thin layer of a viscous liquid, Izv. Akad. Nauk SSSR, Mekh. Zhidk. Gaza 2 (1968) 20-26.

[37] Y.Y. Trifonov, O.Y. Tsvelodub, Nonlinear waves on the surface of a falling liquid film. Part 1. Waves of the first family and their stability, J. Fluid Mech. 229 (1991) 531-554.

[38] Y.Y. Trifonov, O.Y. Tsvelodub, Nonlinear waves on the surface of a falling liquid film. Part 2. Bifurcations of the first-family waves and other types of non-linear waves, J. Fluid Mech. 244 (1992) 149-169.
[39] B. Scheid, C. Ruyer-Quil, P. Manneville, Wave patterns in film flows: modelling and three dimensional waves, J. Fluid Mech. 562 (2006) 183-222.

[40] D. Bessems, M. Rutten, F. van de Vosse, A wave propagation model of blood flow in large vessels using an approximate velocity profile function, J. Fluid Mech. 580 (2007) 145-168.

[41] L. Brevdo, P. Laure, F. Dias, T.J. Bridges, Linear pulse structure and signalling in a film flow on an inclined plane, J. Fluid Mech. 396 (1999) 37-71.

[42] J.C. Guneratne, T.J. Pedley, High-Reynolds-number steady flow in a collapsible channel, J. Fluid Mech. 569 (2006) 151-184.

[43] J. Guckenheimer, P. Holmes, Nonlinear Oscillations, Dynamical Systems and Bifurcations of Vector Fields, Springer, New York, 1983.

[44] D.E. Ashpis, E. Reshotko, The vibrating ribbon problem revisited, J. Fluid Mech. 213 (1990) 531-547.

[45] O. Doaré, E. de Langre, The role of boundary conditions in the instability of one-dimensional systems, Eur. J. Mech. B/Fluids 25 (2006) 948-959.

[46] N. Peake, On the unsteady motion of a long fluid-loaded elastic plate with mean flow, J. Fluid Mech. 507 (2004) 335-366.

[47] P.W. Carpenter, A.D. Garrad, The hydrodynamic stability of flow over Kramertype compliant surfaces. Part 1. Tollmien-Schlichting instabilities, J. Fluid Mech. 155 (1985) 465-510.

[48] P.W. Carpenter, A.D. Garrad, The hydrodynamic stability of flow over Kramertype compliant surfaces. Part 2. Flow-induced surface instabilities, J. Fluid Mech. 170 (1986) 199-232.

[49] F. Gallaire, J.-M. Chomaz, The role of boundary conditions in a simple model of incipient vortex breakdown, Phys. Fluids 16 (2) (2004) 274-286.

[50] D. Serre, Matrices: Theory and Applications, Springer, New York, 2002.

[51] O. Wiplier, U. Ehrenstein, On the absolute instability in a boundary layer with compliant coatings, Eur. J. Mech. B/Fluids (2001) 127-144.

[52] O. Doaré, E. de Langre, Local and global stability of fluid-conveying pipes on elastic foundations, J. Fluids Struct. 16 (1) (2002) 1-14 